\title{
INTEGRAL FIELD SPECTROSCOPY AND MULTI-WAVELENGTH IMAGING OF THE NEARBY SPIRAL GALAXY NGC 5668*: AN UNUSUAL FLATTENING IN METALLICITY GRADIENT
}

\author{
R. A. Marino ${ }^{1,2}$, A. Gil de Paz ${ }^{1}$, A. Castillo-Morales ${ }^{1}$, J. C. Muñoz-Mateos ${ }^{3}$, S. F. Sánchez ${ }^{2}$, P. G. Pérez-González ${ }^{1,6}$, \\ J. Gallego ${ }^{1}$, J. Zamorano ${ }^{1}$, A. Alonso-Herrero ${ }^{4}$, And S. Boissier ${ }^{5}$ \\ ${ }^{1}$ CEI Campus Moncloa, UCM-UPM, Departamento de Astrofísica y CC. de la Atmósfera, Facultad de CC. Físicas, Universidad Complutense de Madrid, \\ Avda. Complutense s/n, 28040 Madrid, Spain; ramarino@ fis.ucm.es \\ ${ }^{2}$ Centro Astronómico Hispano Alemán, Calar Alto (CSIC-MPG), C/Jesús Durbán Remón 2-2, E-04004 Almeria, Spain \\ ${ }^{3}$ National Radio Astronomy Observatory, 520 Edgemont Road, Charlottesville, VA 22903-2475, USA \\ ${ }^{4}$ Instituto de Fisica de Cantabria, CSIC-UC, Avenida de los Castros s/n, 39005 Santander, Spain \\ ${ }^{5}$ Laboratoire dÁstrophysique de Marseille, OAMP, Université Aix-Marseille \& CNRS UMR 6110, 38 rue Frédéric Joliot-Curie, 13388 Marseille cedex 13, France \\ Received 2012 January 10; accepted 2012 May 18; published 2012 July 6
}

\begin{abstract}
We present an analysis of the full bidimensional optical spectral cube of the nearby spiral galaxy NGC 5668, observed with the Pmas fiber PAcK Integral Field Unit (IFU) at the Calar Alto observatory $3.5 \mathrm{~m}$ telescope. We make use of broadband imaging to provide further constraints on the evolutionary history of the galaxy. This data set will allow us to improve our understanding of the mechanisms that drive the evolution of disks. We investigated the properties of $62 \mathrm{H}$ II regions and concentric rings in NGC 5668 and derived maps in ionized-gas attenuation and chemical (oxygen) abundances. We find that while inward of $r \sim 36^{\prime \prime} \sim 4.4 \mathrm{kpc} \sim 0.36\left(D_{25} / 2\right)$ the derived $\mathrm{O} / \mathrm{H}$ ratio follows the radial gradient typical of spiral galaxies, the abundance gradient beyond $r \sim 36^{\prime \prime}$ flattens out. The analysis of the multi-wavelength surface brightness profiles of NGC 5668 is performed by fitting these profiles with those predicted by chemo-spectrophotometric evolutionary models of galaxy disks. From this, we infer a spin and circular velocity of $\lambda=0.053$ and $v_{c}=167 \mathrm{~km} \mathrm{~s}^{-1}$, respectively. The metallicity gradient and rotation curve predicted by this best-fitting galaxy model nicely match the values derived from the IFU observations, especially within $r \sim 36^{\prime \prime}$. The same is true for the colors despite some small offsets and a reddening in the bluest colors beyond that radius. On the other hand, deviations of some of these properties in the outer disk indicate that a secondary mechanism, possibly gas transfer induced by the presence of a young bar, must have played a role in shaping the recent chemical and star formation histories of NGC 5668.
\end{abstract}

Key words: galaxies: abundances - galaxies: evolution - galaxies: individual (NGC 5668) - galaxies: ISM galaxies: kinematics and dynamics - techniques: spectroscopic

Online-only material: color figures

\section{INTRODUCTION}

The evolution of galactic disks is one of the most important and yet not fully understood topics in extragalactic astronomy. Despite significant progress in the recent past regarding our understanding of the history of both thick and thin disks, important questions remain unanswered: How old are the disks seen in the spiral galaxies today? How did they chemically evolve? Are they growing inside-out, as proposed to explain the color and metallicity gradients of the Milky Way (MW)? Do they have an edge? How efficient is stellar radial diffusion?

Until recently, the study of the properties of spiral disks has been limited to broadband imaging data and/or long-slit spectroscopy, which has severely limited the reach of previous works. Radial color profiles have been widely used to probe the inside-out scenario of disk evolution (Bell \& de Jong 2000; MacArthur et al. 2004; Pohlen \& Trujillo 2006; Muñoz-Mateos et al. 2007). However, radial variations of metallicity and dust extinction conspire to create color gradients similar to those due to changes in stellar age alone. Analysis of color-magnitude diagrams (CMDs) allows for more robust measurements of

\footnotetext{
* Based on observations collected at the German-Spanish Astronomical Center, Calar Alto, jointly operated by the Max-Planck-Institut für Astronomie Heidelberg and the Instituto de Astrofísica de Andalucía (CSIC).

6 Associate Astronomer at Steward Observatory, University of Arizona, AZ, USA.
}

stellar ages as a function of radius, but these kinds of studies are currently limited to very nearby galaxies where individual stars can be resolved (de Jong et al. 2007; Barker et al. 2010; Gogarten et al. 2010). Long-slit spectra overcome the problems of broadband photometry, but at the expense of focusing along a pre-defined spatial axis (Yoachim \& Dalcanton 2008). Our effort is committed to adding another dimension for studying nearby spiral galaxies thanks to the use of wide-field Integral Field Spectrometers (IFS) combined with images covering a wide wavelength range from the ultraviolet to the infrared. This paper presents an analysis of the Integral Field Unit (IFU) data taken for NGC 5668 within a more ambitious project aimed at mapping a sample of a dozen nearby galaxies included in the Herschel Galaxy Reference Survey (Boselli et al. 2010) using mosaics obtained with two of the largest IFUs available to date: the Pmas fiber PAcK (PPAK) IFU of the Potsdam MultiAperture Spectrophotometer (PMAS) at the Calar Alto (CAHA) $3.5 \mathrm{~m}$ telescope and the VIsible MultiObject Spectrograph at the ESO Very Large Telescope.

The evolution of galaxy disks is a complex process because many factors are mechanisms that might alter their photometric, chemical, and kinematical properties: minor mergers, the formation of bars or rings, density waves, stellar diffusion, gas infall, and, in the case of galaxies in clusters, also ram pressure stripping and galaxy harassment. Despite this rather discouraging scenario, a picture for the formation of disks has 

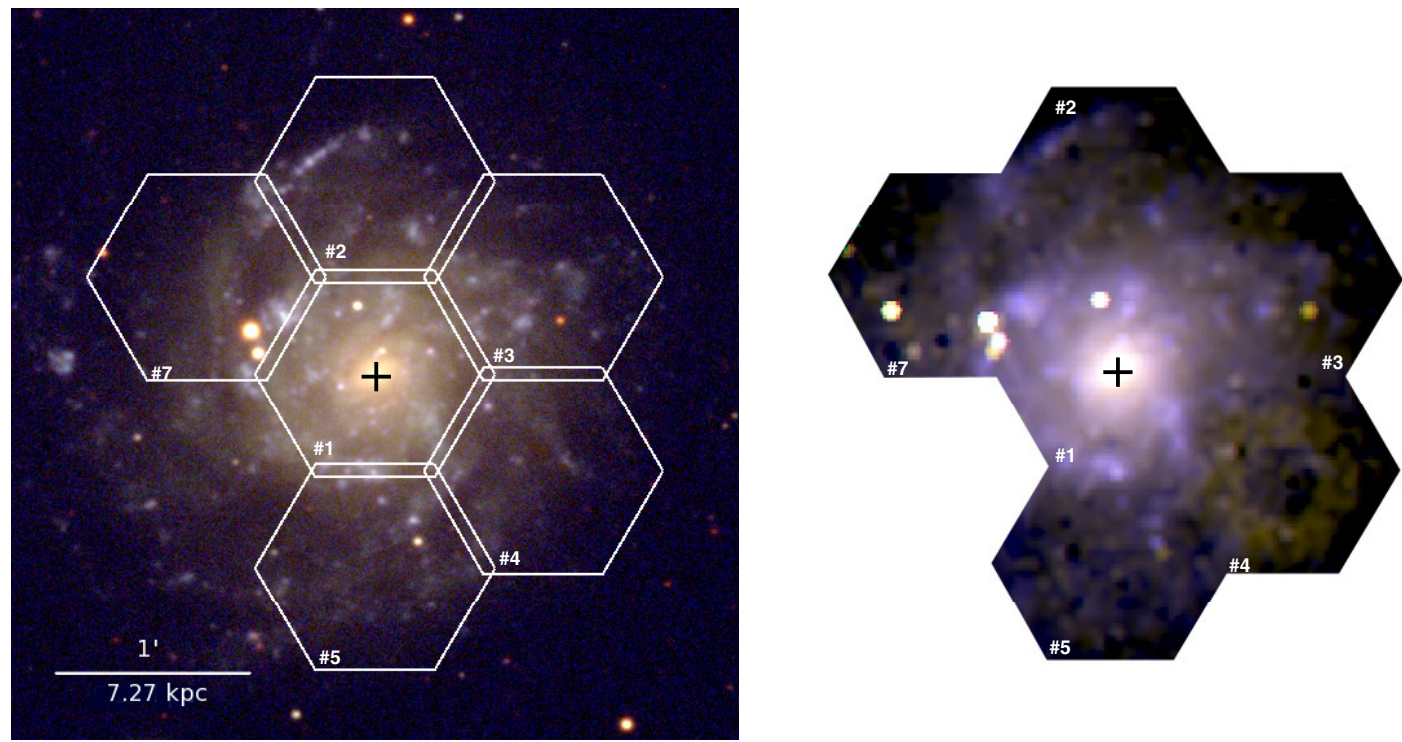

Figure 1. Left: SDSS optical ugr image (from 4458 to $7706 \AA$ ) of NGC 5668 centered at R.A.(2000) $14^{\mathrm{h}} 33^{\mathrm{m}} 24^{\mathrm{s}} 3$ and decl.(2000) $+04^{\circ} 33^{\prime} 24^{\prime \prime} \cdot 3$. North is up and east is to the left. Plate scale is $0.396^{\prime \prime}$ pixel $^{-1}$. Right: synthetic false-color image obtained from the PPAK data cube and the response curves of the SDSS $u, g$, and $r$ filters. Note that the luminous spot in pointing 7 is caused by cross-talk effects from a nearby (on the CCD) field star. Plate scale is $1^{\prime \prime}$ pixel ${ }^{-1}$ in this case. Center and orientation are the same as in the left panel.

emerged in recent years that attempts to explain most of their observational properties: the inside-out formation scenario. Although this scenario has been around for a long time now, the inside-out growth of galaxy disks has only recently been applied and quantified beyond the MW. Both the extinction-corrected color gradients in nearby galaxies (Muñoz-Mateos et al. 2007) and weak dependence of the mass-size relation with redshift (Trujillo et al. 2004, 2006; Barden et al. 2005) support an inside-out scenario for the evolution of disks. However, there are several observational results that conflict with or, at least, cannot be explained by this otherwise elegant scenario. In this regard, many nearby spiral disks show that while the color and metallicity gradients of the inner disk are compatible with the inside-out scenario, beyond the star formation threshold radius (Martin \& Kennicutt 2001) the gradients flatten and even reverse by getting redder toward the outer regions (Bakos et al. 2008; Azzollini et al. 2008; Vlajić et al. 2009). In the specific case of the chemical abundances, the models based on the standard inside-out scenario of galaxy disk formation predict a relatively quick self-enrichment with oxygen abundances one-tenth the solar value after only 1 Gyr of evolution (Boissier \& Prantzos 1999). These models also predict an almost universal negative metallicity gradient once this is normalized to the galaxy optical size $d[\mathrm{O} / \mathrm{H}]$ of $\sim-0.8 \mathrm{dex} / R_{25}$ (Prantzos \& Boissier 2000). Regarding this, the few observational abundance measurements obtained to date in the outer edges of disks show a flattening or even an increase in the abundance toward the outermost parts of the disk. This is also true in the case of the extended UV disks recently discovered by the Galaxy Evolution Explorer (GALEX) satellite (Gil de Paz et al. 2005, 2007; Thilker et al. 2007), which show oxygen abundances that are rarely below one-tenth the solar value. In this regard, Bresolin et al. (2009a) found that the normalized radial metallicity gradient in M83 changes from -0.030 dex $\mathrm{kpc}^{-1}$ in the inner disk to $-0.005 \mathrm{dex} \mathrm{kpc}^{-1}$ in the outer parts (see also Bresolin et al. 2012). We also refer the reader to Yoachim et al. (2010) for another recent example in this same regard. Similar results were obtained on the metallicity gradient of the outer disk of NGC 300 from single-star CMD analysis (Vlajić et al. 2009).
In this work, we present the pilot study of the full bidimensional spectral cube of the nearby spiral galaxy NGC 5668 (Figure 1), obtained with the PPAK IFU at the CAHA observatory $3.5 \mathrm{~m}$ telescope. Despite the relatively modest collecting area of the CAHA $3.5 \mathrm{~m}$ telescope, the broad spectral coverage (from 3700 to $7000 \AA$ ) and adequate spectral resolution $(R=$ $500)$ of PPAK with the V300 grating make this instrument one of the best tools for studying stellar populations, dust content, and physical conditions of the gas (temperature, density, and chemical abundances) in spatially resolved galaxies.

\section{THE NEARBY SPIRAL GALAXY NGC 5668}

NGC 5668 is a nearly face-on late-type spiral galaxy classified as an Sc(s)II-III type according to Sandage \& Tammann (1987) and as an SA(s)d by de Vaucouleurs et al. (1991). There is a weak bar or oval inner structure $12^{\prime \prime}$ in size visible on the optical image, which leads to a small shoulder in the surface brightness profile published by Schulman et al. (1994). The outer disk (beyond $R=100^{\prime \prime}$ ) is slightly asymmetric and more extended toward the north. For this work, we adopted a distance of $\sim 25 \mathrm{Mpc},(m-M)=31.99 \mathrm{mag}$, assuming a cosmology with $H_{0}=73 \mathrm{~km} \mathrm{~s}^{-1} \mathrm{Mpc}^{-1}, \Omega_{\text {matter }}=0.27$, and $\Omega_{\text {vacuum }}=$ 0.73 , for a recession velocity of $1813 \mathrm{~km} \mathrm{~s}^{-1}$ corrected to the reference frame defined by the $3 \mathrm{~K}$ microwave background radiation (Fixsen et al. 1996). ${ }^{7}$ A broadband optical image of this galaxy from the Sloan Digital Sky Survey (SDSS; York et al. 2000 ) is shown in Figure 1. The galaxy presents an inclination of $18^{\circ}$ and a total magnitude of $B=12.13 \pm 0.03$ mag (Schulman et al. 1996). The total mass within the optical disk was estimated to be $5.7 \times 10^{10} M_{\odot}$ (Schulman et al. 1996). ${ }^{8}$ NGC 5668 has been found to host a number of High-velocity Clouds (HVCs) by Schulman et al. (1996) using the Arecibo telescope. These authors detected high-velocity wings in the line shape, which were attributed to HVCs in the galaxy. HVCs and High Residual

\footnotetext{
Source: NASA/IPAC Extragalactic Database (http://nedwww.ipac.caltech.edu/)

8 They adopted a distance of $30 \mathrm{Mpc}$, consistent with a cosmology with $H_{0}=$ $50 \mathrm{~km} \mathrm{~s}^{-1} \mathrm{Mpc}^{-1}$.
} 
Table 1

Global Properties of NGC 5668

\begin{tabular}{|c|c|c|}
\hline Name & NGC 5668 & Source \\
\hline \multirow[t]{2}{*}{ Morphological type } & $\mathrm{SA}(\mathrm{s}) \mathrm{d}$ & de Vaucouleurs et al. (1991) \\
\hline & Sc(s)II-III & Sandage \& Tammann (1987) \\
\hline R.A.(2000) & $14^{\mathrm{h}} 33^{\mathrm{m}} 24^{\mathrm{s}} .3$ & Dressel \& Condon (1976) \\
\hline Decl.(2000) & $+04^{\circ} 27^{\prime} 02^{\prime \prime}$ & Dressel \& Condon (1976) \\
\hline$B$ & $12.13 \pm 0.03 \mathrm{mag}$ & Schulman (1996) \\
\hline$R$ & $11.28 \pm 0.01 \mathrm{mag}$ & Schulman (1996) \\
\hline$L_{B}$ & $(2.7 \pm 0.6) \times 10^{9} L_{\odot}^{a}$ & Schulman (1996) \\
\hline$L_{\mathrm{H} \alpha}$ & $(1.0 \pm 0.3) \times 10^{8} L_{\odot}^{\mathrm{a}}$ & Schulman (1996) \\
\hline$L_{\mathrm{FIR}}$ & $(5.8 \pm 1.2) \times 10^{9} L_{\odot}^{\mathrm{a}}$ & Schulman (1996) \\
\hline$D_{25}$ & $3.3 \pm 0.2$ & Jiménez-Vicente \& Battaner (2000) \\
\hline Heliocentric systemic velocity & $1582 \pm 5 \mathrm{~km} \mathrm{~s}^{-1}$ & Schulman (1996) \\
\hline Distance & $24.8 \pm 1.7 \mathrm{Mpc}$ & Fixsen (1996) \\
\hline Inclination & $18^{\circ}$ & Schulman (1996) \\
\hline P.A. & $145^{\circ}$ & Schulman (1996) \\
\hline Total dynamical mass & $5.7 \times 10^{10} M_{\odot}$ & Schulman (1996) \\
\hline$E(B-V)$ & $0.037 \mathrm{mag}$ & NED \\
\hline
\end{tabular}

Note. ${ }^{\mathrm{a}}$ Corrected for Galactic and internal extinction.

Velocity Regions (residual velocity field and the shell/chimney candidates, regions which have a systematic deviation from rotational velocity) were found also in the ionized gas and interpreted as regions with vertical motions related to ongoing star-forming processes in the disk on the basis of Fabry-Perot $\mathrm{H} \alpha$ observations (Jiménez-Vicente \& Battaner 2000). Schulman et al. (1996) also computed $L_{\mathrm{FIR}}=(5.8 \pm 1.2) \times 10^{9} L_{\odot}$ and $L_{\mathrm{H} \alpha}=(1.0 \pm 0.3) \times 10^{8} L_{\odot}$.

Such high FIR and $\mathrm{H} \alpha$ luminosities (and therefore star formation rates (SFRs)) would naturally result in a high supernova (SN) rate in this galaxy. Indeed, NGC 5668 is known as an "SNe factory" due to the discovery of multiple SN explosions in the recent epoch, namely, SN2004G (Nakano et al. 2004), and SN1952G, SN1954B (Boffi et al. 1999). The main properties of NGC 5668 are summarized in Table 1. NGC 5668 has also been recently observed by a number of instruments and facilities, including SAURON at the William Herschel Telescope (WHT), SDSS, Spitzer and as a part of the Medium-deep Imaging Survey of GALEX. This data set in combination with the PPAK mosaic obtained as part of this work should allow a very detailed analysis of the evolution and chemical-enrichment history of NGC 5668.

\section{OBSERVATIONS AND DATA REDUCTION}

\subsection{IFS Observations}

We have observed the nearly face-on spiral galaxy NGC 5668 with the PPAK IFU of PMAS at the CAHA observatory $3.5 \mathrm{~m}$ telescope (Kelz et al. 2006). The observations were carried out on 2007 June 22-24. We used the PPAK mode that yields a total field of view of $74^{\prime \prime} \times 65^{\prime \prime}$ (hexagonal packed) for each pointing. We covered a total area of roughly $2 \times 3 \mathrm{arcmin}^{2}$ with a mosaic of six PPAK pointings (see Figure 1). We used for the V300 grating covering a wavelength range of 3700-7100 $(10 \AA$ FWHM, corresponding to $\sigma \sim 300 \mathrm{~km} \mathrm{~s}^{-1}$ at $\mathrm{H} \beta$ ). With this spectral configuration, we covered all the optical strong emission lines in which we are interested. A total of 112 individual images were taken during the observing run. These images include 19 on-source frames for the 6 pointings obtained in NGC 5668 (see Table 2 for a summary of the science observations), bias frames (22 in total), sky flats (19), focus images (6), $\mathrm{HgCdHe}$ arcs (14),
Table 2

NGC 5668 Observational Log

\begin{tabular}{lccccc}
\hline \hline Pointing $^{\text {a }}$ & $\begin{array}{c}\text { Obs. Date } \\
(\mathrm{UT})\end{array}$ & $\begin{array}{c}\text { Offsets } \\
(\operatorname{arcsec})\end{array}$ & $\begin{array}{c}\text { Exposure Time } \\
(\mathrm{s})\end{array}$ & Air Mass & $\begin{array}{c}\text { Seeing } \\
(\operatorname{arcsec}) \\
(1)\end{array}$ \\
\hline 1 & $(3)$ & $(4)$ & $(5)$ & $(6)$ \\
\hline 2 & 2007 Jun 22 & $(0,0)$ & $3 \times 1000$ & 1.21 & 1.1 \\
3 & 2007 Jun 22 & $(0,60)$ & $4 \times 1000$ & 1.38 & 1.1 \\
7 & 2007 Jun 22/23 & $(52,60)$ & $3 \times 1000$ & 1.75 & 1.1 \\
5 & 2007 Jun 23 & $(52,-60)$ & $3 \times 1000$ & 1.21 & 1.2 \\
4 & 2007 Jun 23 & $(0,-60)$ & $3 \times 1000$ & 1.36 & 1.2 \\
\hline
\end{tabular}

Notes. (1) NGC 5668 pointings. (2) Observation date. (3) Pointing offsets; the original pointing of the telescope is at R.A.(2000) $14^{\mathrm{h}} 33^{\mathrm{m}} 24^{\mathrm{s}} 3$ and decl.(2000) $+04^{\circ} 33^{\prime} 24^{\prime \prime}$. 3. (4) Exposure time in seconds and the number of images obtained for each pointing. (5) and (6) Mean air mass and seeing values.

a Pointing number 6 in the default mapping strategy used with PPAK at the CAHA observatory has offsets $\left(-52^{\prime \prime},-60^{\prime \prime}\right)$ relative to the center of the mosaic and was not observed in the case of NGC 5668.

tungsten-lamp flats (14), observations of the spectrophotometric standard star Hz44 (3), and dome flats (6). The spatial sampling is determined by a hexagonal array of 331 densely packed optical fibers for the object (science fibers), 36 fibers for the sky, and 15 calibration fibers. Each fiber has a diameter of 2".68 and a pitch of 3".458 with a high filling factor in one single pointing $(65 \%)$. For a more exhaustive description of the instrument, see Kelz et al. (2006).

\subsection{IFS Data Reduction}

The reduction procedure applied to NGC 5668 follows the techniques described in Sánchez (2006) using R3D. This is a software package developed specifically for the reduction of fiber-based IFS data, which has been extensively used for the reduction of PMAS data, in combination with IRAF $^{9}$ packages and Euro3D software (Sánchez 2004). Six pointings were observed for NCG 5668, leading to total covered area of

\footnotetext{
9 IRAF is distributed by the National Optical Astronomy Observatory, which is operated by the Association of Universities for Research in Astronomy, Inc. under cooperative agreement with the National Science Foundation.
} 
$2 \times 3 \operatorname{arcmin}^{2}$. Each pointing results from the combination of three or four images, each one with an exposure time of $1000 \mathrm{~s}$. Our combined raw mosaic includes a total of 2292 spectra (1982+310 science+internal calibration spectra) covering the spectral range $3700-7131 \AA$. The pre-reduction of the IFS data consists of all standard corrections applied to the CCD that are common to the reduction of any CCD-based data.

1. Bias subtraction. A master bias frame was created by averaging all the bias frames observed during the night and subtracted from the science frames.

2. Cosmic-ray rejection. We median combined different exposures (at least three) of the same pointing. This procedure is able to remove $95 \%$ of all cosmic-ray events from each pointing.

After these corrections, the IFS data reduction consists of

1. Spectra extraction. We identified the position of the spectra on the detector for each pixel along the dispersion axis. We then co-added the flux within an aperture of 5 pixels around the trace of the spectra and we extracted the flux corresponding to the different spectra at each pixel along the dispersion axis. Finally, the one-dimensional extracted spectra are stored in a row-staked-spectra (RSS) file.

2. Wavelength calibration. We corrected for all distortions and determined the wavelength solution by using an arc calibration lamp exposure. Calibration data cubes were taken at the beginning and at the end of the night by illuminating the instrument with an $\mathrm{HgCdHe}$ lamp. The V300 wavelength calibration was performed using 14 lines in the considered spectral range. The rms of the best-fitting polynomial (order 4) was $0.45 \AA$ (i.e., FWHM/20).

3. Fiber-to-fiber transmission. We corrected the differences in fiber-to-fiber transmission by using twilight sky exposures from which a Fiber-Flat frame was computed.

4. Sky emission subtraction. For each pointing, a sky image of $300 \mathrm{~s}$ ( shifted $+5^{\prime}$ in decl.) was taken. We median combined the different sky images to create a sky frame, which was then subtracted from the science data frame.

5. Relative flux calibration. We relative flux calibrated our data using observations of the spectrophotometric standard star Hz44 $\left(\alpha(2000)=13^{\mathrm{h}} 23^{\mathrm{m}} 35^{\mathrm{s}} \cdot 37, \delta(2000)=+36^{\circ} 08^{\prime} 00^{\prime} \cdot 0\right.$, Oke 1990) obtained during the night with the same instrumental setup as for the object. We applied all of the previous steps to the calibration star frame and extracted the standard star spectrum from the brightest 2 ".7 wide PPAK fiber centered on the star. Finally, we derived the ratio between counts per second and flux. In this way we obtained the instrumental response, which was applied to the science frames in order to flux calibrate them. Note that although this procedure also provides an absolute calibration for the data, this is uncertain due to potential flux loses during the observation of the standard star and variations in the transparency throughout the night.

6. Differential atmospheric refraction (DAR) correction. We calculated a total theoretical DAR value using a Calar Alto public tool, obtaining a value of $1^{\prime \prime}$ (the mean value of air mass used is 1.3). In this case the DAR can be considered negligible compared with the fiber size ( 2 . 7 in diameter). Therefore, we considered the DAR correction not to be required for this data cube. In any case, we never obtained any flux measurements from regions with less than five adjacent fibers in size.
7. Locating the spectra in the sky. A position table that relates each spectrum to a certain fiber gives the location of the spectra in the sky.

8. Mosaic reconstruction. We built a single RSS file for the whole mosaic by adding pointings previously normalized to a reference ("master") pointing. The "master" pointing is that having the best sky subtraction and most optimal observing conditions regardless of the geometric position of the pointing in the mosaic. In our case this "master" pointing was number 5 (offsets $(0,-60)$; see Figure 1). This procedure makes use of the overlapping fibers between different pointings (11 fibers in total) to re-scale each new added pointing using the flux ratio in the continuum region 5869-5893 $\AA$ between the two pointings (the newly added and the "master" one).

9. Absolute flux re-calibration. We also computed an absolute flux re-calibration based on SDSS photometry. Only two of the five filters of SDSS ( $g$ and $r, \lambda_{\text {eff }}=4694$ and $6178 \AA$, respectively) are used because these are the ones for which their passbands are fully covered by our spectra. We convolved the whole data cube of NGC 5668 with the SDSS $g$ - and $r$-filter passbands to derive the absolute spectrophotometry. First, we measured the AB magnitude within the footprint of the entire PPAK mosaic and in seven concentric annuli for both PPAK and SDSS images. Then, these magnitudes were converted to fluxes using the prescription in the SDSS documentation ${ }^{10}$ in order to calculate the flux ratio in both bands. The resulting average scaling factor found is 0.9 for the two data pairs. This result implies a factor of 0.04 in magnitude. Note that the error in SDSS photometry alone is estimated to be $2 \%$ mag. In our case, we have an uncertainty of $5 \%$ in fluxes, so the total absolute error associated with line fluxes is $8 \%$. Obviously, relative measurements are more precise. All results shown in the following analysis (and in Table 3) are re-calibrated based on these SDSS photometry measurements.

\subsection{Imaging Observations}

In order to complement the IFS data of NGC 5668 presented here, we have also compiled multi-wavelength broadband images acquired with different facilities: UV imaging data from GALEX, optical data from SDSS, and near-IR data from Spitzer (see Figure 2). The GALEX telescope (Martin et al. 2005) observed NGC 5668 as part of its Medium-deep Imaging Survey. Images at both the FUV ( $\left.\lambda_{\text {eff }}=151.6 \mathrm{~nm}\right)$ and NUV $\left(\lambda_{\text {eff }}=226.7 \mathrm{~nm}\right)$ bands are available for this galaxy. The final pixel scale is $1^{\prime \prime} .5$ pixel $^{-1}$, and the point-spread function (PSF) has an FWHM of $\sim 5^{\prime \prime}-6^{\prime \prime}$ in both bands (Morrissey et al. 2007). At the distance of NGC 5668, this corresponds to a physical scale of $\sim 700 \mathrm{pc}$. The uncertainty of the zero point, which is calibrated against white dwarfs, is estimated to be less than 0.15 mag (Gil de Paz et al. 2007).

Optical images in the ugriz bands were retrieved from the SDSS (York et al. 2000) Data Release 7 (DR7) archive (Abazajian et al. 2009). The two different scans on which NGC 5668 lies were flux-matched and mosaicked together using a custombuilt task in IRAF. We applied the photometric calibration specified in the SDSS DR7 Flux Calibration Guide, ${ }^{11}$ relying on the calibration factors of the scan used as reference when creating the mosaic. According to the information provided by the

\footnotetext{
10 http://www.sdss3.org/dr8/algorithms/fluxcal.php

11 http://www.sdss.org/dr7/algorithms/fluxcal.html
} 
Table 3

Spectra Emission Lines

\begin{tabular}{|c|c|c|c|c|c|c|c|}
\hline $\begin{array}{l}\text { ID } \\
\text { (1) }\end{array}$ & $\begin{array}{c}F_{[\mathrm{O}}{ }_{\text {II }} / F_{\mathrm{H} \alpha} \\
(2)\end{array}$ & $\underset{(3)}{F_{\left[\mathrm{O}_{\mathrm{III}}\right]} / F_{\mathrm{H} \alpha}}$ & 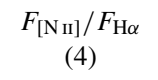 & $\underset{\text { (5) }}{F_{[\mathrm{S} \text { на }]} / F_{\mathrm{H} \alpha}}$ & $\underset{(6)}{F_{[\mathrm{S} \mathrm{IIb]}]} / F_{\mathrm{H} \alpha}}$ & $\begin{array}{l}F_{\mathrm{H} \alpha} \\
(7)\end{array}$ & $\begin{array}{l}A_{V} \\
(8)\end{array}$ \\
\hline \multicolumn{8}{|c|}{ Rings } \\
\hline 1 & & $\ldots$ & . & & & & \\
\hline 2 & $2.68 \pm 0.43$ & $\ldots$ & $0.32 \pm 0.05$ & $0.28 \pm 0.05$ & $0.27 \pm 0.05$ & $18.20 \pm 2.51$ & 1.57 \\
\hline 3 & $1.92 \pm 0.31$ & $0.15 \pm 0.04$ & $0.31 \pm 0.05$ & $0.26 \pm 0.04$ & $0.24 \pm 0.04$ & $19.40 \pm 2.41$ & 1.52 \\
\hline 4 & $1.77 \pm 0.29$ & $0.21 \pm 0.05$ & $0.26 \pm 0.04$ & $0.25 \pm 0.04$ & $0.20 \pm 0.04$ & $13.94 \pm 1.77$ & 1.23 \\
\hline 5 & $1.13 \pm 0.21$ & $0.21 \pm 0.05$ & $0.29 \pm 0.06$ & $0.25 \pm 0.06$ & $0.23 \pm 0.05$ & $4.96 \pm 0.71$ & 0.35 \\
\hline 6 & $1.70 \pm 0.32$ & $0.28 \pm 0.06$ & $0.26 \pm 0.05$ & $0.25 \pm 0.05$ & $0.19 \pm 0.05$ & $9.36 \pm 1.41$ & 1.00 \\
\hline 7 & $1.83 \pm 0.24$ & $0.36 \pm 0.05$ & $0.21 \pm 0.03$ & $0.23 \pm 0.03$ & $0.18 \pm 0.03$ & $10.61 \pm 1.18$ & 1.33 \\
\hline 8 & $1.30 \pm 0.18$ & $0.40 \pm 0.06$ & $0.17 \pm 0.03$ & $0.21 \pm 0.03$ & $0.17 \pm 0.03$ & $7.12 \pm 0.78$ & 0.88 \\
\hline 9 & $2.29 \pm 0.41$ & $0.47 \pm 0.09$ & $0.20 \pm 0.05$ & $0.24 \pm 0.05$ & $0.25 \pm 0.05$ & $7.86 \pm 1.29$ & 1.64 \\
\hline 10 & $2.04 \pm 0.48$ & $0.61 \pm 0.14$ & $0.15 \pm 0.06$ & $0.23 \pm 0.07$ & $0.19 \pm 0.06$ & $4.36 \pm 0.93$ & 1.33 \\
\hline 11 & $1.81 \pm 0.58$ & $0.35 \pm 0.13$ & $\ldots$ & $0.21 \pm 0.09$ & $0.21 \pm 0.09$ & $2.80 \pm 0.80$ & 1.19 \\
\hline 12 & $\ldots$ & $\ldots$ & $\ldots$ & $\ldots$ & $\ldots$ & $\ldots$ & $\ldots$ \\
\hline 13 & $\ldots$ & $\ldots$ & $\ldots$ & $\ldots$ & $\ldots$ & $\ldots$ & $\ldots$ \\
\hline 14 & $1.99 \pm 0.87$ & $0.64 \pm 0.28$ & $\ldots$ & $\ldots$ & $\ldots$ & $1.80 \pm 0.75$ & 1.10 \\
\hline 15 & $1.50 \pm 0.43$ & $0.66 \pm 0.18$ & $\ldots$ & $\ldots$ & $\ldots$ & $2.03 \pm 0.53$ & 0.76 \\
\hline \multicolumn{8}{|c|}{ H II regions } \\
\hline 1 & $1.14 \pm 0.14$ & $0.64 \pm 0.04$ & $0.09 \pm 0.02$ & $0.28 \pm 0.03$ & $0.19 \pm 0.03$ & $2.23 \pm 0.09$ & 0.65 \\
\hline 2 & $1.19 \pm 0.37$ & $0.48 \pm 0.11$ & $0.20 \pm 0.09$ & $\ldots$ & $\ldots$ & $0.50 \pm 0.05$ & 0.12 \\
\hline 3 & $1.55 \pm 0.23$ & $0.49 \pm 0.04$ & $0.15 \pm 0.02$ & $0.24 \pm 0.02$ & $0.13 \pm 0.01$ & $11.36 \pm 0.28$ & 2.02 \\
\hline 4 & $1.52 \pm 0.09$ & $0.24 \pm 0.02$ & $0.16 \pm 0.01$ & $0.21 \pm 0.01$ & $0.17 \pm 0.01$ & $12.27 \pm 0.16$ & 1.36 \\
\hline 5 & $1.33 \pm 0.06$ & $0.37 \pm 0.01$ & $0.17 \pm 0.01$ & $0.22 \pm 0.01$ & $0.15 \pm 0.01$ & $13.32 \pm 0.14$ & 1.18 \\
\hline 6 & $\ldots$ & $\ldots$ & $0.01 \pm 0.01$ & $0.28 \pm 0.05$ & $0.25 \pm 0.05$ & $5.39 \pm 0.43$ & 2.46 \\
\hline 7 & $1.83 \pm 0.42$ & $0.44 \pm 0.08$ & $0.02 \pm 0.01$ & $0.28 \pm 0.05$ & $0.13 \pm 0.03$ & $3.68 \pm 0.21$ & 1.64 \\
\hline 8 & $1.44 \pm 0.12$ & $0.29 \pm 0.04$ & $0.09 \pm 0.03$ & $0.18 \pm 0.03$ & $0.21 \pm 0.04$ & $11.39 \pm 0.50$ & 1.31 \\
\hline 9 & $1.57 \pm 0.11$ & $0.46 \pm 0.03$ & $0.12 \pm 0.02$ & $0.12 \pm 0.02$ & $0.10 \pm 0.02$ & $32.00 \pm 0.91$ & 2.06 \\
\hline 10 & $1.72 \pm 0.07$ & $0.41 \pm 0.01$ & $0.17 \pm 0.01$ & $0.17 \pm 0.01$ & $0.13 \pm 0.01$ & $12.39 \pm 0.16$ & 1.22 \\
\hline 11 & $2.00 \pm 0.22$ & $0.62 \pm 0.05$ & $0.14 \pm 0.02$ & $0.26 \pm 0.02$ & $0.20 \pm 0.02$ & $4.75 \pm 0.18$ & 1.30 \\
\hline 12 & $2.05 \pm 0.26$ & $0.67 \pm 0.04$ & $0.11 \pm 0.02$ & $0.24 \pm 0.02$ & $0.14 \pm 0.02$ & $10.02 \pm 0.30$ & 1.98 \\
\hline 13 & $2.31 \pm 0.23$ & $0.34 \pm 0.06$ & $0.18 \pm 0.05$ & $0.26 \pm 0.06$ & $0.14 \pm 0.05$ & $4.27 \pm 0.25$ & 0.39 \\
\hline 14 & $1.76 \pm 0.80$ & $0.32 \pm 0.10$ & $0.20 \pm 0.05$ & $0.16 \pm 0.06$ & $\ldots$ & $23.48 \pm 1.85$ & 2.60 \\
\hline 15 & $0.48 \pm 0.08$ & $0.57 \pm 0.05$ & $0.10 \pm 0.04$ & $0.15 \pm 0.05$ & $0.12 \pm 0.04$ & $6.27 \pm 0.25$ & 0.73 \\
\hline 16 & $1.18 \pm 0.09$ & $0.47 \pm 0.02$ & $0.15 \pm 0.01$ & $0.17 \pm 0.01$ & $0.11 \pm 0.01$ & $15.94 \pm 0.23$ & 1.61 \\
\hline 17 & $3.93 \pm 0.76$ & $0.96 \pm 0.16$ & $0.18 \pm 0.07$ & $0.22 \pm 0.06$ & $0.22 \pm 0.09$ & $19.32 \pm 1.93$ & 2.89 \\
\hline 18 & $1.26 \pm 0.37$ & $\ldots$ & $0.22 \pm 0.03$ & $0.29 \pm 0.03$ & $0.13 \pm 0.03$ & $6.84 \pm 0.30$ & 1.93 \\
\hline 19 & $1.48 \pm 0.17$ & $0.29 \pm 0.04$ & $0.26 \pm 0.03$ & $0.27 \pm 0.03$ & $0.18 \pm 0.03$ & $11.43 \pm 0.50$ & 0.90 \\
\hline 20 & $0.51 \pm 0.12$ & $0.41 \pm 0.04$ & $0.25 \pm 0.03$ & $0.28 \pm 0.04$ & $0.17 \pm 0.03$ & $1.375 \pm 0.05$ & 0.12 \\
\hline 21 & $1.85 \pm 0.14$ & $0.29 \pm 0.03$ & $0.22 \pm 0.02$ & $0.15 \pm 0.02$ & $0.11 \pm 0.02$ & $16.73 \pm 0.59$ & 0.89 \\
\hline 22 & $2.40 \pm 0.62$ & $0.26 \pm 0.10$ & $0.18 \pm 0.05$ & $0.35 \pm 0.05$ & $0.24 \pm 0.04$ & $4.30 \pm 0.34$ & 1.87 \\
\hline 23 & $0.93 \pm 0.04$ & $0.97 \pm 0.01$ & $0.09 \pm 0.01$ & $0.12 \pm 0.01$ & $0.10 \pm 0.01$ & $11.94 \pm 0.12$ & 0.92 \\
\hline 24 & $1.69 \pm 0.15$ & $0.26 \pm 0.03$ & $0.23 \pm 0.02$ & $0.22 \pm 0.02$ & $0.16 \pm 0.02$ & $7.36 \pm 0.18$ & 1.31 \\
\hline 25 & $1.57 \pm 0.27$ & $0.71 \pm 0.07$ & $0.24 \pm 0.04$ & $0.14 \pm 0.03$ & $0.10 \pm 0.03$ & $2.41 \pm 0.12$ & 1.06 \\
\hline 26 & $1.41 \pm 0.51$ & $0.14 \pm 0.05$ & $0.31 \pm 0.07$ & $0.33 \pm 0.07$ & $0.21 \pm 0.05$ & $9.14 \pm 1.68$ & 0.88 \\
\hline 27 & $\ldots$ & $2.19 \pm 0.48$ & $\ldots$ & $0.15 \pm 0.07$ & $\ldots$ & $31.30 \pm 4.64$ & 4.59 \\
\hline 28 & $1.74 \pm 0.47$ & $0.17 \pm 0.05$ & $0.30 \pm 0.05$ & $0.26 \pm 0.04$ & $0.19 \pm 0.04$ & $20.57 \pm 2.59$ & 1.67 \\
\hline 29 & $1.28 \pm 0.20$ & $0.21 \pm 0.03$ & $0.25 \pm 0.03$ & $0.24 \pm 0.03$ & $0.19 \pm 0.03$ & $14.89 \pm 0.93$ & 1.08 \\
\hline 30 & $1.34 \pm 0.24$ & $0.24 \pm 0.05$ & $0.25 \pm 0.04$ & $0.25 \pm 0.04$ & $0.16 \pm 0.03$ & $11.61 \pm 0.71$ & 1.15 \\
\hline 31 & $1.30 \pm 0.08$ & $0.38 \pm 0.02$ & $0.18 \pm 0.01$ & $0.18 \pm 0.01$ & $0.11 \pm 0.01$ & $9.23 \pm 0.12$ & 1.14 \\
\hline 32 & $1.13 \pm 0.09$ & $0.33 \pm 0.02$ & $0.18 \pm 0.01$ & $0.15 \pm 0.01$ & $0.11 \pm 0.01$ & $29.66 \pm 0.59$ & 1.18 \\
\hline 33 & $1.43 \pm 0.13$ & $0.20 \pm 0.02$ & $0.24 \pm 0.02$ & $0.19 \pm 0.02$ & $0.12 \pm 0.01$ & $30.84 \pm 1.21$ & 1.30 \\
\hline 34 & $2.30 \pm 0.59$ & $0.41 \pm 0.10$ & $0.14 \pm 0.04$ & $0.24 \pm 0.03$ & $0.13 \pm 0.03$ & $6.59 \pm 0.41$ & 2.20 \\
\hline 35 & $3.42 \pm 0.38$ & $0.39 \pm 0.06$ & $0.35 \pm 0.04$ & $0.35 \pm 0.04$ & $0.21 \pm 0.04$ & $50.93 \pm 3.45$ & 1.50 \\
\hline 36 & $2.32 \pm 0.15$ & $0.66 \pm 0.03$ & $0.14 \pm 0.01$ & $0.19 \pm 0.01$ & $0.12 \pm 0.01$ & $17.41 \pm 0.34$ & 1.96 \\
\hline 37 & $1.15 \pm 0.05$ & $0.36 \pm 0.01$ & $0.18 \pm 0.01$ & $0.14 \pm 0.01$ & $0.11 \pm 0.01$ & $57.29 \pm 1.04$ & 1.00 \\
\hline 38 & $1.55 \pm 0.23$ & $0.15 \pm 0.04$ & $0.26 \pm 0.03$ & $0.24 \pm 0.03$ & $0.15 \pm 0.03$ & $13.94 \pm 0.79$ & 1.25 \\
\hline 39 & $2.68 \pm 0.92$ & $0.96 \pm 0.14$ & $\ldots$ & $\ldots$ & $\ldots$ & $7.80 \pm 0.59$ & 2.74 \\
\hline 40 & $3.32 \pm 1.16$ & $0.51 \pm 0.11$ & $0.15 \pm 0.03$ & $0.26 \pm 0.03$ & $0.09 \pm 0.02$ & $25.61 \pm 1.71$ & 3.63 \\
\hline 41 & $1.06 \pm 0.05$ & $0.32 \pm 0.01$ & $0.16 \pm 0.01$ & $0.19 \pm 0.01$ & $0.15 \pm 0.01$ & $14.14 \pm 0.16$ & 1.06 \\
\hline 42 & $2.21 \pm 0.45$ & $0.27 \pm 0.07$ & $0.24 \pm 0.05$ & $0.23 \pm 0.04$ & $0.20 \pm 0.04$ & $7.85 \pm 1.30$ & 1.84 \\
\hline 43 & $0.76 \pm 0.16$ & $0.20 \pm 0.03$ & $0.22 \pm 0.03$ & $0.23 \pm 0.03$ & $0.18 \pm 0.03$ & $18.11 \pm 1.75$ & 1.22 \\
\hline 44 & $1.78 \pm 0.19$ & $0.15 \pm 0.03$ & $0.32 \pm 0.03$ & $0.23 \pm 0.02$ & $0.24 \pm 0.02$ & $28.78 \pm 2.28$ & 1.47 \\
\hline 45 & $2.99 \pm 0.41$ & $0.12 \pm 0.04$ & $0.29 \pm 0.04$ & $0.27 \pm 0.04$ & $0.21 \pm 0.03$ & $25.34 \pm 3.02$ & 1.75 \\
\hline 46 & $1.33 \pm 0.25$ & $0.15 \pm 0.05$ & $0.25 \pm 0.05$ & $0.26 \pm 0.05$ & $0.21 \pm 0.04$ & $11.02 \pm 1.50$ & 1.06 \\
\hline 47 & $1.45 \pm 0.20$ & $0.16 \pm 0.04$ & $0.25 \pm 0.04$ & $0.26 \pm 0.04$ & $0.21 \pm 0.04$ & $6.23 \pm 0.71$ & 0.34 \\
\hline
\end{tabular}


Table 3

(Continued)

\begin{tabular}{|c|c|c|c|c|c|c|c|}
\hline $\begin{array}{l}\text { ID } \\
(1)\end{array}$ & $\begin{array}{c}F_{[\mathrm{O} \text { II }]} / F_{\mathrm{H} \alpha} \\
\text { (2) }\end{array}$ & $\begin{array}{c}F_{[\mathrm{OIII}]} / F_{\mathrm{H} \alpha} \\
\text { (3) }\end{array}$ & $\begin{array}{c}F_{[\mathrm{N} \text { II }]} / F_{\mathrm{H} \alpha} \\
\text { (4) }\end{array}$ & $\begin{array}{c}F_{\left[\mathrm{S}_{\text {IIa }}\right]} / F_{\mathrm{H} \alpha} \\
(5)\end{array}$ & $\begin{array}{c}F_{\left[\mathrm{S}_{\text {IIb }]}\right]} / F_{\mathrm{H} \alpha} \\
(6)\end{array}$ & $\begin{array}{c}F_{\mathrm{H} \alpha} \\
(7)\end{array}$ & $\begin{array}{l}A_{V} \\
(8)\end{array}$ \\
\hline 48 & $3.60 \pm 0.75$ & $0.26 \pm 0.08$ & $0.26 \pm 0.06$ & $0.29 \pm 0.07$ & $0.18 \pm 0.05$ & $15.20 \pm 2.89$ & 1.71 \\
\hline 49 & $1.64 \pm 0.05$ & $0.75 \pm 0.01$ & $0.10 \pm 0.01$ & $0.11 \pm 0.01$ & $0.10 \pm 0.01$ & $16.48 \pm 0.16$ & 1.09 \\
\hline 50 & $1.22 \pm 0.19$ & $0.20 \pm 0.04$ & $0.25 \pm 0.04$ & $0.25 \pm 0.04$ & $0.21 \pm 0.04$ & $9.11 \pm 1.05$ & 0.72 \\
\hline 51 & $1.56 \pm 0.11$ & $0.34 \pm 0.03$ & $0.21 \pm 0.02$ & $0.20 \pm 0.02$ & $0.17 \pm 0.02$ & $22.45 \pm 1.29$ & 0.93 \\
\hline 52 & $1.14 \pm 0.11$ & $0.31 \pm 0.03$ & $0.22 \pm 0.02$ & $0.22 \pm 0.02$ & $0.19 \pm 0.02$ & $21.20 \pm 1.41$ & 1.01 \\
\hline 53 & $1.52 \pm 0.05$ & $0.40 \pm 0.01$ & $0.18 \pm 0.01$ & $0.19 \pm 0.01$ & $0.16 \pm 0.01$ & $29.32 \pm 0.54$ & 1.02 \\
\hline 54 & $1.65 \pm 0.06$ & $0.66 \pm 0.02$ & $0.12 \pm 0.01$ & $0.15 \pm 0.01$ & $0.12 \pm 0.01$ & $10.30 \pm 0.12$ & 0.94 \\
\hline 55 & $1.72 \pm 0.08$ & $0.63 \pm 0.02$ & $0.12 \pm 0.01$ & $0.22 \pm 0.01$ & $0.16 \pm 0.01$ & $15.11 \pm 0.18$ & 1.33 \\
\hline 56 & $0.81 \pm 0.05$ & $0.18 \pm 0.02$ & $0.11 \pm 0.02$ & $0.24 \pm 0.02$ & $0.16 \pm 0.02$ & $3.30 \pm 0.16$ & 0.00 \\
\hline 57 & $11.5 \pm 2.4$ & $0.78 \pm 0.16$ & $0.18 \pm 0.04$ & $0.18 \pm 0.04$ & $0.16 \pm 0.04$ & $153.29 \pm 30.27$ & 3.93 \\
\hline 58 & $1.83 \pm 0.08$ & $0.70 \pm 0.02$ & $0.11 \pm 0.02$ & $0.15 \pm 0.02$ & $0.13 \pm 0.03$ & $6.07 \pm 0.16$ & 0.86 \\
\hline 59 & $3.22 \pm 0.25$ & $0.45 \pm 0.04$ & $0.13 \pm 0.04$ & $0.19 \pm 0.03$ & $0.27 \pm 0.04$ & $6.09 \pm 0.27$ & 1.51 \\
\hline 60 & $2.26 \pm 0.14$ & $0.36 \pm 0.03$ & $0.10 \pm 0.03$ & $0.17 \pm 0.03$ & $0.16 \pm 0.04$ & $4.80 \pm 0.18$ & 1.06 \\
\hline 61 & $7.2 \pm 1.3$ & $0.78 \pm 0.11$ & $\ldots$ & $0.17 \pm 0.05$ & $0.21 \pm 0.07$ & $20.34 \pm 1.59$ & 3.57 \\
\hline 62 & $3.24 \pm 0.61$ & $\ldots$ & $0.28 \pm 0.05$ & $0.25 \pm 0.05$ & $0.21 \pm 0.04$ & $34.30 \pm 5.43$ & 2.30 \\
\hline Total $^{\mathrm{a}}$ & $1.31 \pm 0.17$ & $0.35 \pm 0.05$ & $0.16 \pm 0.04$ & $0.21 \pm 0.03$ & $0.15 \pm 0.03$ & $3.93 \pm 0.14$ & 1.03 \\
\hline
\end{tabular}

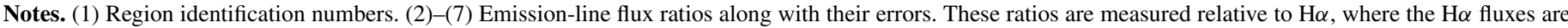

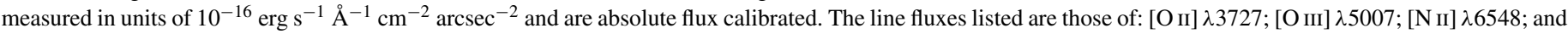

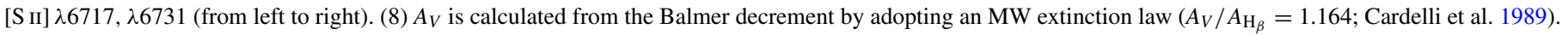
a The term total refers to the integrated spectrum. In this case, the flux line ratio values are in units of $10^{-12} \mathrm{erg} \mathrm{s}^{-1} \mathrm{~cm}^{-2}$.
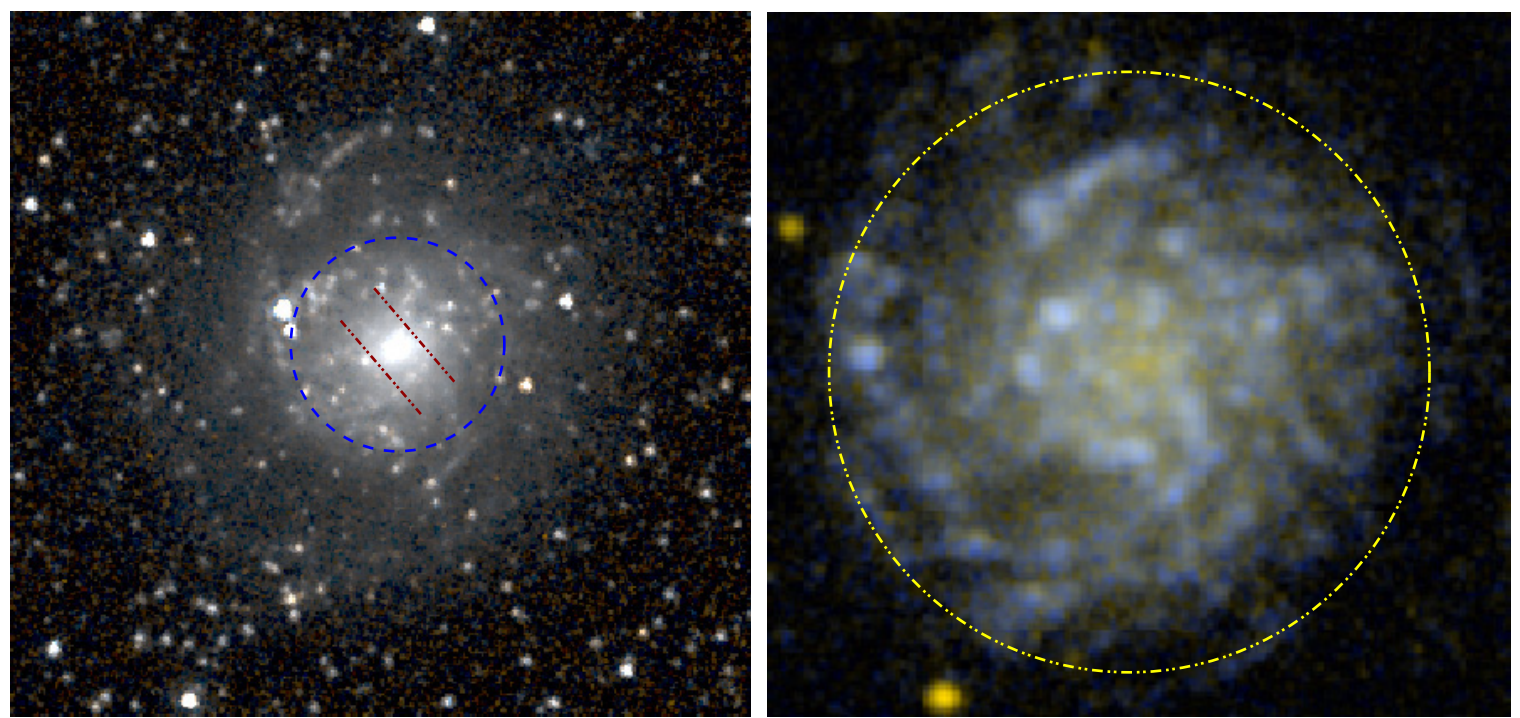

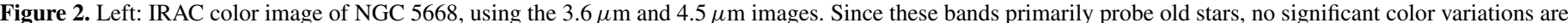

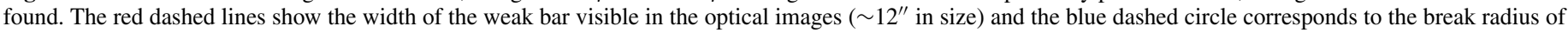

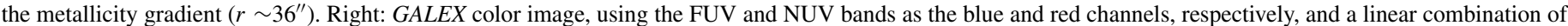

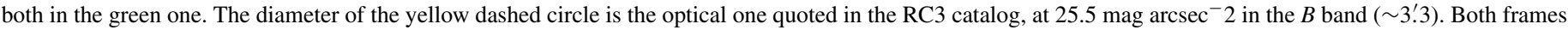
are $250^{\prime \prime}$ in size. North is up and east is to the left.

SDSS project, the photometric relative errors are of the order of $2 \%-3 \%$. The plate scale is 0.396 pixel $^{-1}$ with a PSF FWHM of 1 ". 4 (170 pc at the distance of the galaxy).

Near-IR images at $3.6 \mu \mathrm{m}$ and $4.5 \mu \mathrm{m}$ were taken by Spitzer (Werner et al. 2004) using the IRAC instrument (Fazio et al. 2004). The corresponding Post Basic Calibrated Data were downloaded from the Spitzer archive. ${ }^{12}$ These images are already flux calibrated and delivered in units of $\mathrm{MJy} \mathrm{sr}^{-1}$. The pixel scale is 0.6 pixel $^{-1}$, and the PSF has an FWHM of 1".7 in both channels, probing a spatial extent of $200 \mathrm{pc}$ (Reach et al. 2005). The photometric error $(\sim 2 \%)$ is dominated by the aperture corrections that need to be applied to account for the

\footnotetext{
12 Spitzer Proposal ID 69: A Mid-IR Hubble Atlas of Galaxies, PI: G. Fazio.
}

scattered light on the array. This total uncertainty is estimated to be around $10 \%^{13}$ in this case.

\section{ANALYSIS}

\subsection{Spectroscopy}

Many important topics in astrophysics involve the physics of ionized gases and the interpretation of their emission-line spectra. Powerful constraints on theories of galactic chemical evolution and on the star formation histories (SFHs) of galaxies can be derived from the accurate determination of chemical abundances either in individual star-forming regions or distributed

\footnotetext{
13 http://ssc.spitzer.caltech.edu/irac/calib/extcal/
} 


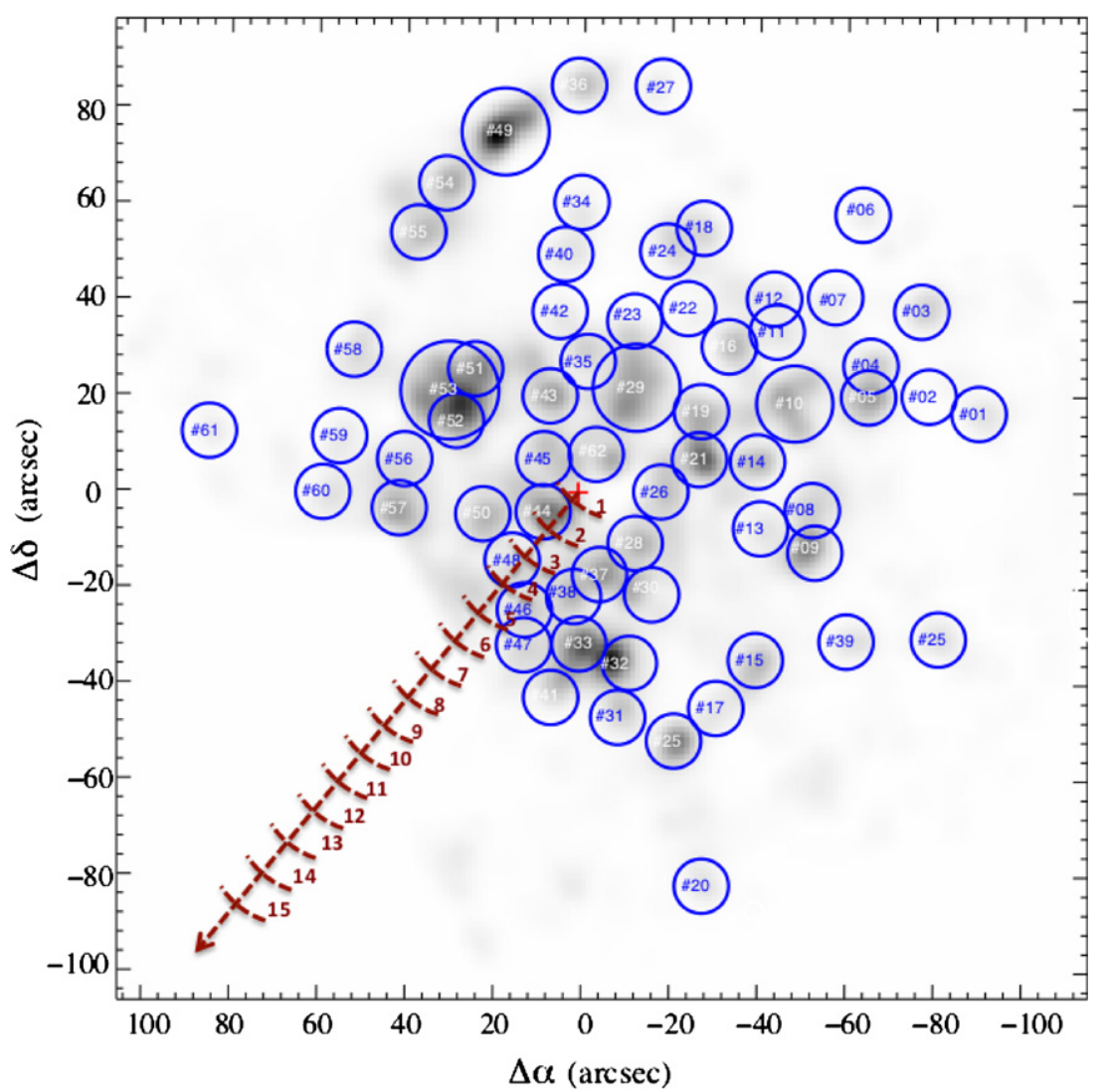

Figure 3. PPAK continuum-subtracted $\mathrm{H} \alpha$ emission map. The H II regions selected in NGC 5668 are shown as blue circles; see Section 4.1 .1 for details. The red dashed lines indicate the radii of the rings and the red cross represents the central fiber of the mosaic. North is up and east is to the left.

(A color version of this figure is available in the online journal.)

across galaxies or even in galaxies as a whole. In this sense, chemical abundance is a fossil record of star formation history (SFH). In addition, the distribution of $\mathrm{H}$ II regions is an excellent tracer of recent massive star formation in spiral galaxies. The previous evolutionary history of the gas and the local ionization, density, and temperature are important features for understanding the physical conditions prevailing in the regions where they are emitted.

For all these reasons, our first aim is to identify $\mathrm{H}$ II regions in our target, or at least H II complexes (see Figure 3 ). ${ }^{14}$ We extract from the PPAK data cube the emission-line fluxes corresponding to each of these regions. Furthermore, we also compute twodimensional maps of the flux of strong emission lines, such as [O II] $\lambda \lambda 3726,3729^{15}$; [O III] $\lambda \lambda 4959,5007 ; \mathrm{H} \beta$; [N II $] \lambda 6548$; $\mathrm{H} \alpha$; [N II] $\lambda 6583$; and [S II] $\lambda \lambda 6717,6731$.

\subsubsection{Region Selection}

In spiral galaxies, $\mathrm{H}$ in regions are strongly concentrated along the spiral arms and in the galactic plane and are the best objects for tracing the structure of spiral arms in galaxies. We are interested in the study of the optical emission lines from $\mathrm{H}$ II regions because they represent the primary means of performing gas-phase diagnostics in galaxies (Pagel 1986; Osterbrock \& Ferland 2006). These data were analyzed using IRAF and E3D (Sánchez 2004) tasks and some custom-made

${ }^{14}$ Hereafter we use the terms "H II regions" and "H II complexes" indistinctly, even although individual $\mathrm{H}$ II regions are not spatially resolved at the physical resolution achieved in our IFS data.

15 The [O II] $\lambda \lambda 3726,3729$ doublet is spectrally unresolved in our data, therefore, and hereafter we will refer to it as $[\mathrm{O}$ II] $\lambda 3727$.
IDL (Interactive Data Language) scripts. In order to select the $\mathrm{H}$ II regions of NGC 5668, we computed a synthetic $\mathrm{H} \alpha$ image (the brightest emission line in the optical spectrum of $\mathrm{H}$ II regions under most physical conditions) from the data cube using E3D. The spectral window for the $\mathrm{H} \alpha$ line was defined as a narrow wavelength range from $6589 \AA$ to $6613 \AA$. Two bands (6457-6553 $\AA$ and 6667-6730 $\AA$ ) close to the $\mathrm{H} \alpha$ line were also extracted for continuum subtraction. We normalized the "continuum" maps to match the width of the $\mathrm{H} \alpha$ intensity map. From the synthetic $\mathrm{H} \alpha$ image, we can then identify $\mathrm{H}$ II regions. We visually selected $62 \mathrm{H}$ II complexes in the galaxy from this image (see Figure 3). The apertures were defined so that they would approximately reach the same surface brightness level in $\mathrm{H} \alpha$. We also imposed the conditions that these apertures would be large enough to include at least five PPAK fibers (in order to minimize atmospheric refraction effects) and that each region would be isolated from bright neighbors. As a result of the conditions imposed, the majority of regions are the sum of five PPAK fibers, except in the case of regions 17, 37, and 62, which are the superposition of one bright and several marginally resolved faint $\mathrm{H}$ II regions, and region 58, which extends $15^{\prime \prime}$ in the SE-NW direction approximately following the spiral pattern of the galaxy's northern arm. See Figure 3 for the positions and sizes of the regions of interest selected for this study. We extracted the spectrum coming from each region by averaging the signal for each fiber belonging to a particular region.

In many spiral galaxies of early and intermediate Hubble type (Sa-Sc), active star formation is organized in a ring-like structure that often contains a large fraction of the entire star formation activity of the galaxy. To investigate this and the ubiquitous 
radial variation of the physical properties in spiral galaxies, we also selected 18 concentric annuli, centered on the peak of the optical-continuum emission. A width of about $5^{\prime \prime}$ proved to be a good compromise in terms of the trade-off between spatial resolution and depth. The outermost ring analyzed is located at $R_{\text {last }}=95^{\prime \prime}$.

In a first step we analyze the data to verify the quality of the spectra extracted in all regions: we identify and eliminate bad fibers (e.g., those placed on bright field stars or affected by cross-talk), and we interpolate over the spectrum at the position of the brightest sky lines to clean out our spectra. We trim the wavelength range in each spectrum to avoid the poor instrumental efficiency at the very edges. This leads to a useful wavelength range of 3702-6997 $\AA$.

\subsubsection{Emission-line Fluxes}

The radiation emitted by each element of volume in a region depends upon the abundance of the elements, determined by the previous evolutionary history of the galaxy, and the local ionization, density, and temperature. The most prominent spectral features are emission lines, many of which are collisionally excited lines. In the V300 grating setup, the main emission lines observed are [O II] $\lambda 3727$ doublet; [O III] $\lambda \lambda 4959,5007 ; \mathrm{H} \beta$; [N II] $\lambda 6548 ; \mathrm{H} \alpha ;[\mathrm{N}$ II $] \lambda 6583$; and [S II] $\lambda \lambda 6717,6731$.

We identified two different types of regions according to the intensity of the continuum: (1) regions where the continuum was bright enough so some absorption features were clearly visible and, therefore, the spectrum of the underlying stellar population could be fit using stellar population synthesis models, and (2) regions with fainter and/or noisier continua where no spectral absorption features could be identified. ${ }^{16}$

We refer to Section 4.1.3 for a description of the procedure followed to fit the underlying continuum in the case of the former regions; here we discuss the procedure followed to derive accurate emission-line fluxes in regions with negligible or noisy continua. Initially, we have identified $73 \mathrm{HII}$ regions in our synthetic $\mathrm{H} \alpha$ image but we excluded some of these regions because of their low signal-to-noise ratio $(\mathrm{S} / \mathrm{N})$ or because they were later identified as bad columns or field stars.

We first masked out the main emission lines and used a thirdorder polynomial function to fit the continuum. We then selected two spectral ranges excluding emission lines in three different wavelength ranges $[\mathrm{O} \mathrm{II}] \lambda 3727, \mathrm{H} \beta$, and $\mathrm{H} \alpha$ regions, and measured the continuum level in each of these ranges. In a second step, we averaged the two continua and subtracted the result from each emission-line spectra in order to obtain a decontaminated flux. We analyzed the spectra using the IRAF package ONEDSPEC and STSDAS. For well-isolated emission lines, such as [O II] $\lambda 3727, \mathrm{H} \delta, \mathrm{H} \gamma,{ }^{17}[\mathrm{O}$ III] $\lambda 5007$, we fit the emission-line profile to a single Gaussian function. For partially blended emission lines, such as the $[\mathrm{N}$ II $] \lambda 6548 ; \mathrm{H} \alpha$; [N II] $\lambda 6583$ triplet, and [S II] $\lambda \lambda 6717,6731$ doublet, we used the task NGAUSSFIT that fits simultaneously multiple Gaussian functions. We calculated the typical $\mathrm{S} / \mathrm{N}$ of our data. In the case of [O II] $\lambda 3727$, the $\mathrm{S} / \mathrm{N}$ ranges are between 3.4 and 23 . In the case of $\mathrm{H} \beta$, we obtain similar $\mathrm{S} / \mathrm{N}$ values, ranging between 2.5

\footnotetext{
16 The limit in the continuum intensity between the two types of spectra was set to $1 \times 10^{-16} \mathrm{erg} \mathrm{s}^{-1} \AA^{-1}$. For those spectra where the continuum was fainter (in either $\mathrm{H} \beta$ or $\mathrm{H} \alpha$ ) than this number, no attempt to carry out a full spectral fitting to the continuum was attempted.

17 Note that we detect these lines only in $23 \mathrm{H}$ il regions, so we do not use $\mathrm{H} \delta$ and $\mathrm{H} \gamma$ to derive physical properties.
}

Table 4

$\mathrm{H} \alpha$ Equivalent Widths in Emission from the Ring Spectra

\begin{tabular}{lccccc}
\hline \hline Ring & $\begin{array}{c}\mathrm{EW}_{\mathrm{H} \alpha}^{\mathrm{em}} \\
(\AA)\end{array}$ & $\begin{array}{c}\text { Radius } \\
\left({ }^{\prime \prime}\right)\end{array}$ & Ring & $\begin{array}{c}\mathrm{EW}_{\mathrm{H} \alpha}^{\mathrm{em}} \\
(\AA)\end{array}$ & $\begin{array}{c}\text { Radius } \\
\left({ }^{\prime \prime}\right)\end{array}$ \\
\hline 1 & $\mathrm{a}$ & 1 & 9 & 38.34 & 40 \\
2 & 8.75 & 5 & 10 & 37.85 & 45 \\
3 & 18.65 & 10 & 11 & 36.5 & 50 \\
4 & 29.32 & 15 & 12 & 24.04 & 55 \\
5 & 25.56 & 20 & 13 & 34.98 & 60 \\
6 & 39.18 & 25 & 14 & 50.18 & 65 \\
7 & 44.97 & 30 & 15 & 100.1 & 70 \\
8 & 44.00 & 35 & 16 & 128.5 & $75^{\mathrm{b}}$ \\
\hline
\end{tabular}

Notes. These values are not corrected for underlying stellar absorption.

${ }^{a}$ No line emission was detected.

${ }^{b}$ In this table, we present the value for ring 16 that it is not shown in any of the other tables in the paper since only the $\mathrm{H} \alpha$ line could be detected in the spectrum and its flux (and EW) measured.

and 23. For the part of our spectra where the $\mathrm{H} \alpha$ emission line is located, the $S / N$ values vary between 6 and 67 .

Before analyzing the data in terms of chemical abundances or SFR, the observed emission-line fluxes must be corrected for various effects. For example, the presence of dust between the zone of the emission and the observer or the possible underlying stellar absorption in the case of the hydrogen recombination lines can alter considerably the intrinsic emission-line fluxes. We correct for underlying stellar absorption in the hydrogen Balmer lines using the values obtained from the analysis of the ring spectra (see Section 4.1.3 for fitting details). These bestfitting equivalent widths in absorption show very little variation with a radius ranging between -1.9 and $-2.3 \AA$ in $\mathrm{H} \beta$ and -1.7 and $-1.8 \AA$ in $\mathrm{H} \alpha$. We apply an average correction of -2 and $-1.7 \AA$, respectively, for $\mathrm{H} \beta$ and $\mathrm{H} \alpha$, to the spectra of the $58 \mathrm{H}$ II regions where the continuum emission was too faint for carrying out a full spectral fitting. The emission fluxes were then corrected for reddening using the $\mathrm{H} \alpha / \mathrm{H} \beta$ Balmer decrements after adopting an intrinsic ratio of 2.86 (Osterbrock \& Ferland 2006). Additionally, we verified that in the case of regions with a high equivalent width in emission (typically no less than $5 \AA$ in $\mathrm{H} \beta$ ), the corrected $\mathrm{H} \beta / \mathrm{H} \gamma$ ratios were consistent with the predictions for the case $\mathrm{B}$ recombination value at a typical $T_{e}$ of $\sim 10^{4} \mathrm{~K}$. In Table 3, we present the results obtained from the analysis of H II regions and concentric annuli in NGC 5668.

Following a similar analysis on a pixel-by-pixel basis, we also generate maps of ionized-gas extinction, radial velocity, emission-line fluxes, and stellar-absorption equivalent width. In Figure 4, we present the maps of the $\mathrm{H} \alpha$ line flux, [O II] $\lambda 3727$ doublet flux, $\mathrm{H} \alpha$ continuum intensity, and the equivalent width (EW). Note that while the $\mathrm{EW}(\mathrm{H} \alpha)$ in emission gets locally higher as we move toward the outer parts of the disk, this is mainly due to the decrease in the intensity of the adjacent underlying ( $R$-band) continuum. Indeed, when we compute the azimuthally averaged $\mathrm{EW}(\mathrm{H} \alpha)$ from the ring spectra, this changes less dramatically, with values ranging from 20 to $50 \AA$ in all cases except for the very outer rings (see Table 4 ). The very high $\mathrm{EW}(\mathrm{H} \alpha)$ values measured in the rings beyond $\sim 70^{\prime \prime}$ are likely due to strong $\mathrm{H} \alpha$ emission associated with the bright $\mathrm{H}$ II complex located in the galaxy Northern spiral arm. Note that in the very outer disk, where the total SFR is low, stochasticity in the number and luminosity of $\mathrm{H}$ II regions might lead to significant fluctuations in the $\operatorname{EW}(\mathrm{H} \alpha)$ compared with values 

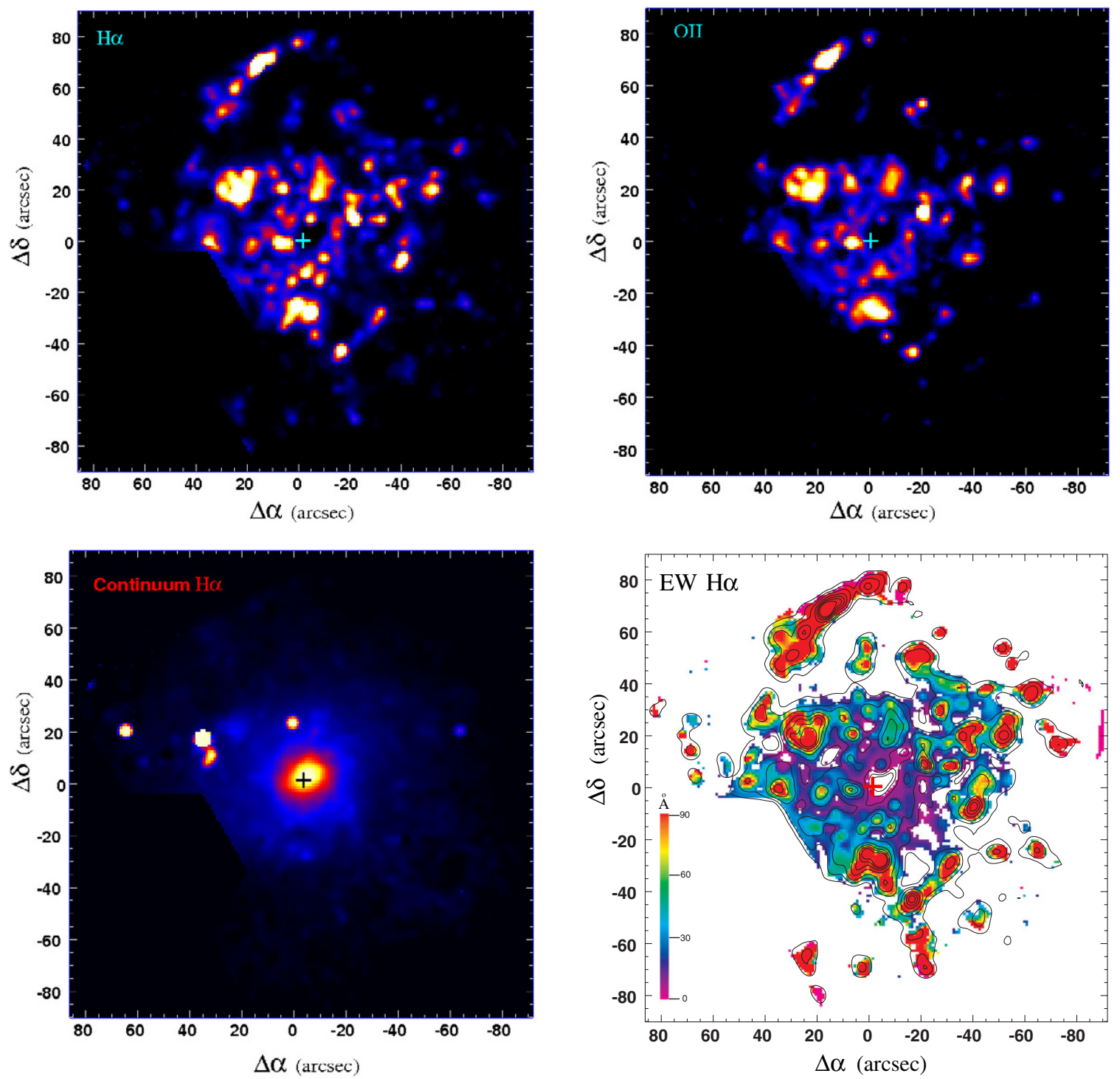

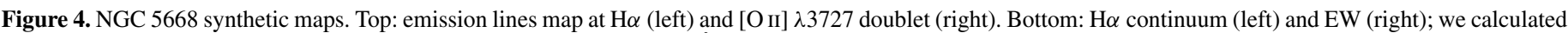

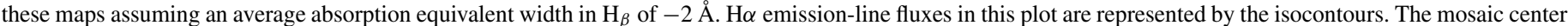
is marked with a cross. North is up and east is to the left in all cases.

(A color version of this figure is available in the online journal.)

averaged over timescales of a few hundred Myr or even a rotation period.

\subsubsection{Stellar-absorption Correction}

As discussed above, for the rings and some $\mathrm{H}$ II regions the continuum is sufficiently strong enough to allow us to clearly identify absorption features in their spectra. In this way we can fit the absorption and emission components in the spectra simultaneously. The spectra of the 18 concentric annuli are plotted in Figure 5. In this case, we made use of an IDL script that finds the best-fitting stellar population synthesis model among the ones in libraries by Bruzual \& Charlot (2003, hereafter BC03) and Sánchez-Blázquez et al. (2006, also known as MILES). This procedure allowed us to measure intensities for the emission lines already corrected for stellar continuum absorption (nebular continuum emission is negligible at the $\mathrm{EW}(\mathrm{H} \alpha)$ measured anywhere in this object). Both libraries yield good fits to our spectra, yet the analysis presented here is based on the results obtained using the BC03 library since this provides a good coverage of physical parameters and good spectral resolution and has been more extensively tested than MILES. We explore the whole range in age and metallicity in the $\mathrm{BC} 03$ library for simple stellar populations (SSPs) with a spectral resolution of $3 \AA$ across the whole wavelength range from $3200 \AA$ to $9500 \AA$. These templates have 11 values in age $(5,25,100,290,640,900 \mathrm{Myr}$, and 1.4, 2.5, 11, 13, $17 \mathrm{Gyr})$ and each of them has six different metallicity values: $z=0.0001$, $0.0004,0.004,0.008,0.02,0.05$. Our fitting program is able to find the model that best fits the underlying stellar population (stellar continuum) in this BC03 stellar library by minimizing the residuals between the model and observed spectra for a set of input parameters. The program finds the best-fitting parameters for the recession velocity, velocity dispersion, normalization factor, and stellar continuum attenuation in the $V$ band for each region.

In order to attenuate the stellar continuum as a function of wavelength, we adopt a dependence of the optical depth with a wavelength of the form $\lambda^{-0.7}$, which, according to the results of 


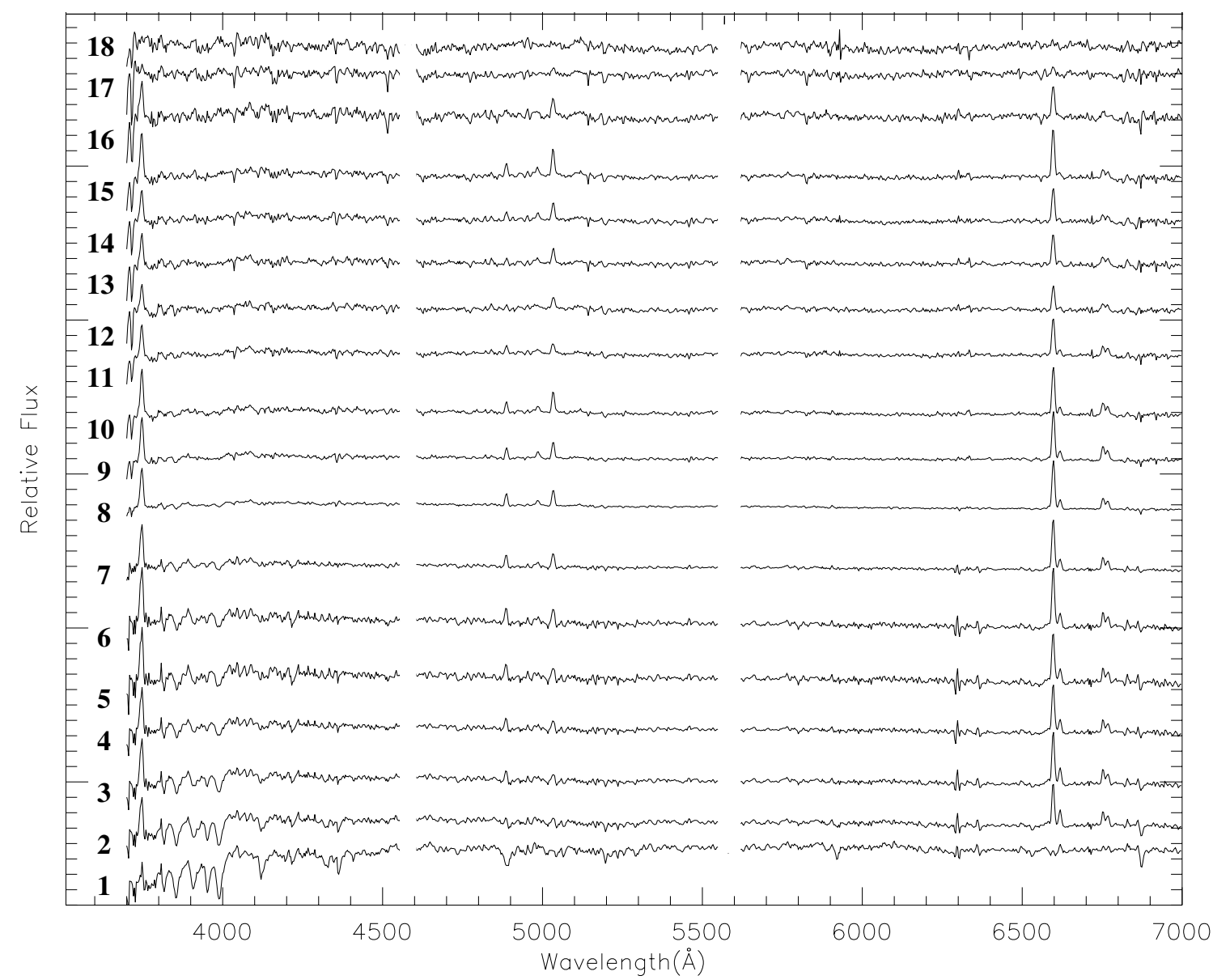

Figure 5. PPAK spectra extracted from the rings selected in NGC 5668. From bottom to top we show the spectra of rings with an increasing radius. The spectra are obtained summing all fibers within each ring. Two bright sky line residual ranges are masked. These spectra are plotted in linear scale and shifted in the $y$-axis for better visualization.

Charlot \& Fall (2000), is a good approximation to the extinction law in star-forming galaxies at these wavelengths. This analysis provides us with the best-fitting SSP model for each spectrum along with best-fitting values for the recession (tropocentric) velocity, velocity dispersion, normalization factor, and continuum dust attenuation.

Note that the age and metallicities derived are, in general, highly uncertain, but, on the other hand, the $\mathrm{H} \alpha$ and $\mathrm{H} \beta$ equivalent widths in absorption are much more precise (see Mármol-Queraltó et al. 2011 for a detailed description on the feasibility in the derivation of the properties of the underlying stellar population). The residual spectrum obtained after the subtraction of the best-fitting SSP model is then used for the subsequent analysis of the emission-line fluxes.

We calculate the errors in all line fluxes from the rms in the spectra (in the region adjacent to each emission feature) after the best-fitting stellar synthesis model has been subtracted. We assume that these rms measurements were due mainly to photon noise and to sky subtraction. These errors were scaled to those spectra for which the fainter continuum emission prevented carrying out a full spectral fitting. Finally, we used the errors in the continuum to calculate the errors associated with the line fluxes. ${ }^{18}$

\footnotetext{
18 The errors are calculated from the residual fit models of the underlying stellar population; we are assuming that these residuals are primarily due to photon noise and sky subtraction. We note that we have pointings with a slightly different spatial resolution and several combinations of exposure time and number of individual exposures; for this reason the continuum level
}

\subsection{Integrated Spectrum}

Some advantages of using IFUs are that all the spectra are obtained simultaneously and that we can use the IFU as a large-aperture spectrograph. From our data cube, we add up the spectra of all the fibers to create an integrated spectrum of NGC 5668 (see Figure 6). In this figure, we also compare this spectrum with the corresponding fluxes derived from the SDSS photometry. Note that the $g^{\prime}$ and $r^{\prime}$ bands overlap completely with our spectral range. Since the spectroscopy data were not taken under photometric conditions and given that some flux losses are expected as part of the PPAK observations of our spectrophotometric standard star, an $8 \%$ difference was found between the two data sets. This offset, which is wavelength independent, has already been applied to the spectrum shown in Figure 6, although it was not applied to our spectral data cube and the line fluxes measured hereafter. Similar wavelengthindependent offsets are also found in other works based on PPAK spectroscopic observations (Rosales-Ortega et al. 2010, 2011). In any case, this does not have any impact on the physical

emission and the associated noise was derived for each pointing separately. The sky subtraction to the spectra of NGC 5668 was done, see the data reduction steps in Sánchez (2006), by median average the 331 sky spectra. The resulting spectrum was then subtracted from the cube of NGC 5668. The analysis of these sky-subtracted spectra showed that the error in the continuum level emission increases linearly with the number of fibers (but not to the square root). This indicates that for apertures including a large number of fibers the total error is dominated by the sky-subtraction error. 


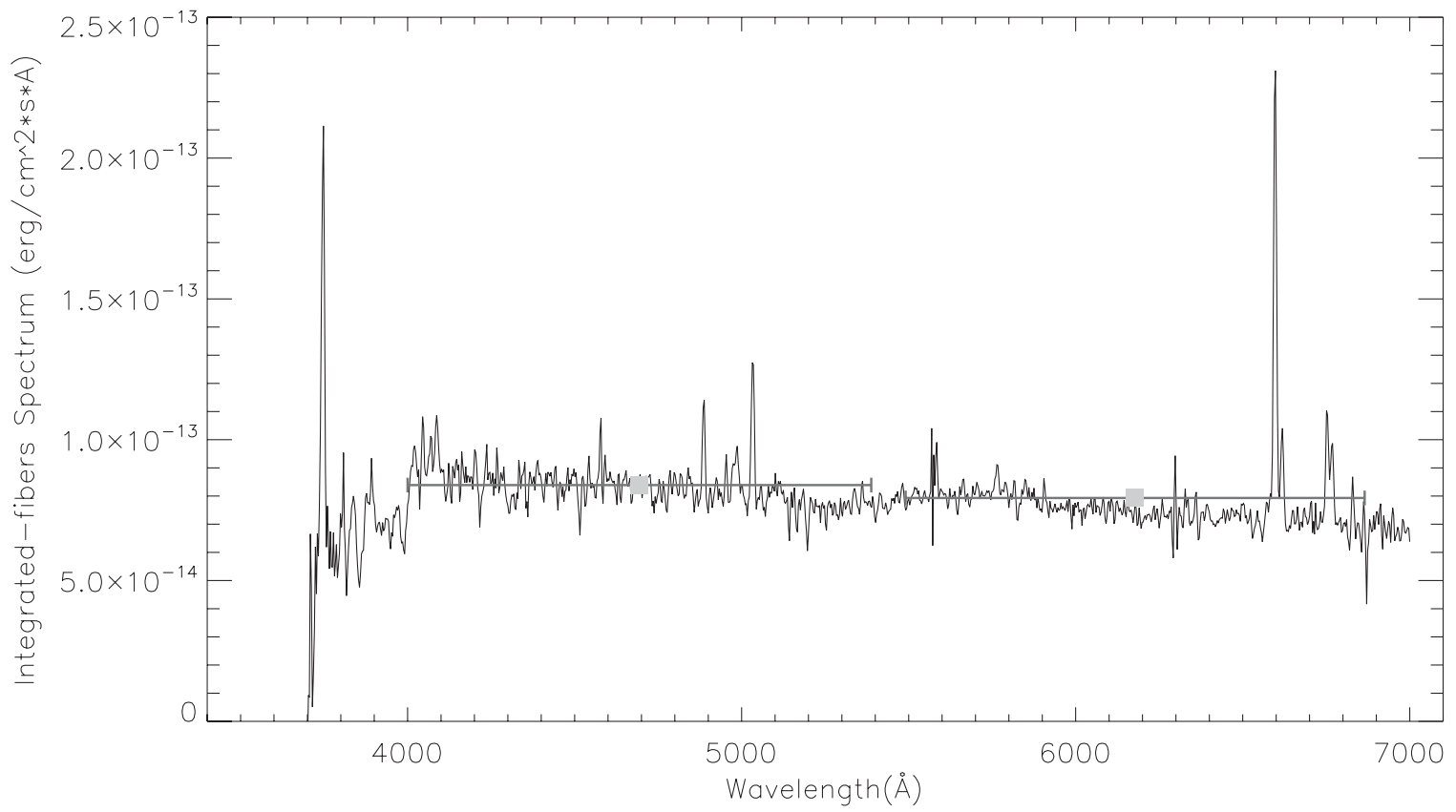

Figure 6. Integrated spectrum of NGC 5668 is shown as a black line. The SDSS $g^{\prime}$ - and $r^{\prime}$-band photometry data are shown as gray squares. Horizontal error bars represent the FWHM of each filter.

properties derived (dust attenuation, oxygen abundance, and electron density) as these are determined by the ratio of emission-line fluxes alone. In Table 3 (last row), we present the emission-line flux ratios found for the integrated fiber spectrum of NGC 5668.

\subsection{Imaging}

Prior to the measurement of the surface brightness profiles in NGC 5668, foreground stars, background galaxies, and cosmetic artifacts had to be masked out from our multi-wavelength imaging data. Radial profiles were obtained using the IRAF task ELLIPSE. We measured the mean intensity within concentric circular annuli centered on the galaxy's nucleus, using a radial increment of $6^{\prime \prime}$ between adjacent annuli. Photometric errors were derived as explained in Gil de Paz \& Madore (2005) and Muñoz-Mateos et al. (2009b). The uncertainty of the mean intensity at a given radius is computed as the quadratic sum of two terms: the Poissonian noise of the galaxy's light and the error in the sky level. The latter was the result of the combination of both pixel-to-pixel variations and large-scale background errors.

\section{RESULTS}

\subsection{Attenuation}

More than 80 years have passed since Trumpler's discovery of color excesses provided the first definitive proof of the existence of interstellar dust (Trumpler 1930). However, the nature of interstellar dust still remains unclear and how dust both reddens and attenuates the light from the stars is one of the least understood of the physical phenomena which take place in galaxies.

The dust content of galaxies is a critical issue given its important impact on the observational properties of galaxies (mainly in the optical and UV). The extinction in a galaxy depends first on the amount of dust and its composition, and also on the distribution of dust relative to the light sources (Calzetti 2001). For instance, some authors favor a foreground screen dust geometry model (Calzetti et al. 1994, 1996), while others propose hybrid models with the dust partially distributed in a foreground screen and partially concentrated in the star-forming regions (Charlot \& Fall 2000). In this subsection, we discuss the methods used to estimate dust attenuation. We calculate the attenuation of the stellar continuum from the UV data available on NGC 5668 and these estimates are then compared with the ionized-gas attenuation derived from the Balmer decrement in both individual $\mathrm{H}$ II regions and concentric annuli.

\subsubsection{UV-continuum Attenuation}

In this case, we estimate the attenuation of the stellar continuum using UV data alone. Because the UV radiation is preferentially emitted by young stars $(\sim 100 \mathrm{Myr})$ and because the dust is most efficient in attenuating UV light, rest-frame UV observations can lead to incomplete and/or biased reconstructions of the recent star formation activity and SFH of galaxies where dust absorption is expected to be significant, unless proper corrections are applied. Radiative transfer models suggest that the total-IR (TIR; 3-1100 $\mu \mathrm{m}$ ) to UV luminosity ratio method (i.e., Buat 1992; Xu \& Buat 1995; Meurer et al. 1995, 1999) is the most reliable estimator of the dust attenuation in star-forming galaxies because it is almost completely independent of the extinction mechanisms (i.e., dust/star geometry, extinction law; see Xu \& Buat 1995; Meurer et al. 1999; Gordon et al. 2000; Witt \& Gordon 2000). However, this would require having both far-infrared data at the same resolution as the UV data. In the case of galaxies at the distance of NGC 5668, this would provide very little spatial information on the radial variation of the dust attenuation even if data from state-of-the-art infrared facilities such as Herschel would be available. Even though IRAC and MIPS images for NGC 5668 are available in the Spitzer archive, and we can theoretically obtain profiles in $8,24,70$, and $160 \mu \mathrm{m}$, the poor resolution at $160 \mu \mathrm{m}\left(38^{\prime \prime}\right)$ would yield a TIR profile with just a couple of data points. Under certain circumstances, the UV attenuation can be indirectly estimated using the slope of the UV spectrum 
Table 5

UV-continuum Attenuation Results

\begin{tabular}{|c|c|c|c|c|c|c|}
\hline $\begin{array}{l}\text { Radius } \\
\left({ }^{\prime \prime}\right) \\
(1)\end{array}$ & $\begin{array}{c}A_{\mathrm{FUV}} \\
\text { (mag) } \\
(2)\end{array}$ & $\begin{array}{c}A_{\mathrm{NUV}} \\
(\mathrm{mag}) \\
(3)\end{array}$ & $\begin{array}{c}E(B-V)_{\text {Cardelli }} \\
(\mathrm{mag}) \\
(4)\end{array}$ & $\begin{array}{l}A_{V, \text { Cardelli }} \\
\quad(\mathrm{mag}) \\
(5)\end{array}$ & $\begin{array}{c}E(B-V) \text { Calzetti } \\
(\mathrm{mag}) \\
(6)\end{array}$ & $\begin{array}{c}A_{V, \text { Calzetti }} \\
(\mathrm{mag}) \\
(7)\end{array}$ \\
\hline 6 & 1.98 & 1.40 & 0.18 & 0.54 & 0.17 & 0.69 \\
\hline 12 & 1.51 & 1.13 & 0.14 & 0.44 & 0.14 & 0.56 \\
\hline 18 & 1.33 & 1.03 & 0.13 & 0.40 & 0.12 & 0.51 \\
\hline 24 & 1.20 & 0.95 & 0.12 & 0.37 & 0.12 & 0.47 \\
\hline 30 & 1.04 & 0.87 & 0.11 & 0.34 & 0.11 & 0.43 \\
\hline 36 & 0.90 & 0.80 & 0.10 & 0.31 & 0.10 & 0.39 \\
\hline 42 & 1.01 & 0.86 & 0.11 & 0.33 & 0.10 & 0.42 \\
\hline 48 & 1.04 & 0.87 & 0.11 & 0.34 & 0.11 & 0.43 \\
\hline 54 & 1.23 & 0.97 & 0.12 & 0.38 & 0.12 & 0.48 \\
\hline 60 & 1.20 & 0.96 & 0.12 & 0.37 & 0.12 & 0.47 \\
\hline 66 & 0.95 & 0.83 & 0.10 & 0.32 & 0.10 & 0.41 \\
\hline 72 & 0.84 & 0.77 & 0.10 & 0.30 & 0.09 & 0.38 \\
\hline 78 & 0.84 & 0.77 & 0.10 & 0.30 & 0.09 & 0.38 \\
\hline 84 & 0.93 & 0.82 & 0.10 & 0.32 & 0.10 & 0.40 \\
\hline 90 & 0.75 & 0.73 & 0.09 & 0.28 & 0.09 & 0.36 \\
\hline 96 & 0.40 & 0.59 & 0.07 & 0.23 & 0.07 & 0.29 \\
\hline 102 & 0.62 & 0.67 & 0.08 & 0.26 & 0.08 & 0.33 \\
\hline 108 & 0.58 & 0.66 & 0.08 & 0.26 & 0.08 & 0.32 \\
\hline 114 & 0.90 & 0.80 & 0.10 & 0.31 & 0.10 & 0.40 \\
\hline
\end{tabular}

Notes. (1) Radius in arcsec. (2) Far-UV attenuation values in magnitudes. (3) Near-UV attenuation values in magnitudes. (4) and (5) Galactic color excess from UV data and attenuation values calculated via Cardelli law, $\left(E(B-V)=A_{\mathrm{NUV}} / 8.0\right) R_{V}=3.1$, expressed in magnitudes. (6) and (7) Galactic color excess from UV data and attenuation values calculated via Calzetti law, $(E(B-V)=$ $\left.A_{\mathrm{NUV}} / 8.22\right) R_{V}=4.05$, expressed in magnitudes.

(denoted as $\beta$ ), in the sense that redder UV colors are indicative of larger attenuations. This so-called infrared excess (IRX)- $\beta$ relation has been widely used in starburst galaxies, where most of the UV light comes from newly born stars (Calzetti et al. 1994; Heckman et al. 1995; Meurer et al. 1999). In normal star-forming spirals, more evolved stars can also contribute to the UV flux, shifting the IRX $-\beta$ relation to redder UV colors and increasing the overall scatter (Bell 2002; Buat et al. 2005; Seibert et al. 2005; Cortese et al. 2006; Gil de Paz et al. 2007; Dale et al. 2007). The use of radial profiles instead of integrated measurements seems to reduce the global scatter (Boissier et al. 2007; Muñoz-Mateos et al. 2009a).

Here, we apply the IRX $-\beta$ relation provided by MuñozMateos et al. (2009a) to FUV-NUV measurements obtained from the GALEX images of NGC 5668. This relationship was specifically calibrated for normal nearby spirals, so it should be applicable to NGC 5668 as well. However, before applying this technique to subregions, we checked this assumption using the total galaxy IR luminosity of NGC 5668 from the IRAS fluxes quoted by Wang \& Rowan-Robinson (2009) leading to a TIR flux of $\log \left(F_{\text {TIR }}\right)=13.64 \times 10^{-23}\left(\mathrm{erg} \mathrm{s}^{-1} \mathrm{~cm}^{-2}\right)$. This value combined with the FUV flux obtained from our GALEX FUV image leads to $\log (\mathrm{TIR} / \mathrm{FUV}) \sim 0.18$. On the other hand, for a global UV color of FUV-NUV $=0.27$, the IRX- $\beta$ yields $\log (\mathrm{TIR} / \mathrm{FUV}) \sim 0.39$. This is somewhat larger than the observed value, but still lies within the $1 \sigma$ scatter around the IRX $-\beta$ relation of Muñoz-Mateos et al. (2009b). Thus, using the radial profiles described in Section 4.3, we determined the radial variation of the FUV-NUV color in steps of $6^{\prime \prime}$. The UV color was then used to obtain profiles of TIR/FUV and TIR/NUV ratios using the prescriptions of Muñoz-Mateos et al. (2009b). These values were then transformed into dust attenuation in the FUV ( $\left.A_{\mathrm{FUV}}\right)$ and NUV ( $\left.A_{\mathrm{NUV}}\right)$ by means of the recipes by Cortese et al. (2008), which take into account the IR excess associated with the extra dust heating due to the light from old stars. The resulting attenuation profiles are shown in Table 5. Note that the absolute uncertainty in the TIR/FUV ratio from the FUV-NUV is larger than the relative change in such a ratio across the galaxy (see Muñoz-Mateos et al. 2009b).

Now that we have derived the extinction in the UV, we can calculate the extinction in other bands using an attenuation curve. Such reddening corrections have normally been undertaken using a number of extinction curves, including those of Seaton (1979; in the UV), Cardelli et al. (1989), Savage \& Mathis (1979), Ardeberg \& Virdefors (1982), and Fitzpatrick (1999). These "standard" curves are often used interchangeably, on the understanding that they should give broadly similar results. The parameterized curve by Cardelli et al. (1989) is commonly used to fit the extinction data both for diffuse and dense interstellar medium (ISM). In our case, the continuum attenuation is obtained using at different wavelengths two different extinction laws (Cardelli et al. 1989; Calzetti 2001). We based our calculation on the NUV data, adopting the relation with the color excess $E(B-V)$ given by Cardelli et al. (1989), $E(B-V)=$ $A_{\mathrm{NUV}} / 8.0$, and for Calzetti et al. (1994), $E(B-V)=A_{\mathrm{NUV}} /$ 8.22 (Gil de Paz et al. 2007). We then adopt the average values of $R_{V, \text { Cardelli }}=3.1$ and $R_{V \text {,Calzetti }}=4.05$. For sake of clarity, Figure 7 plots only the results obtained in the case of the Calzetti extinction law as the two curves are consistent within the errors (but offset by -0.09 mag in the case of the Cardelli law).

\subsubsection{Ionized-gas Emission-line Attenuation}

We now make use of the Balmer decrement in individual $\mathrm{H}$ II regions and concentric annuli for determining the amount of dust extinction in the ionized-gas emission along the line of sight. The relative ratios of the Balmer lines of hydrogen are often used as extinction indicators due to the fact that they are observationally convenient (being in the optical band), strong, and their intrinsic relative flux ratios are fairly well detemined 

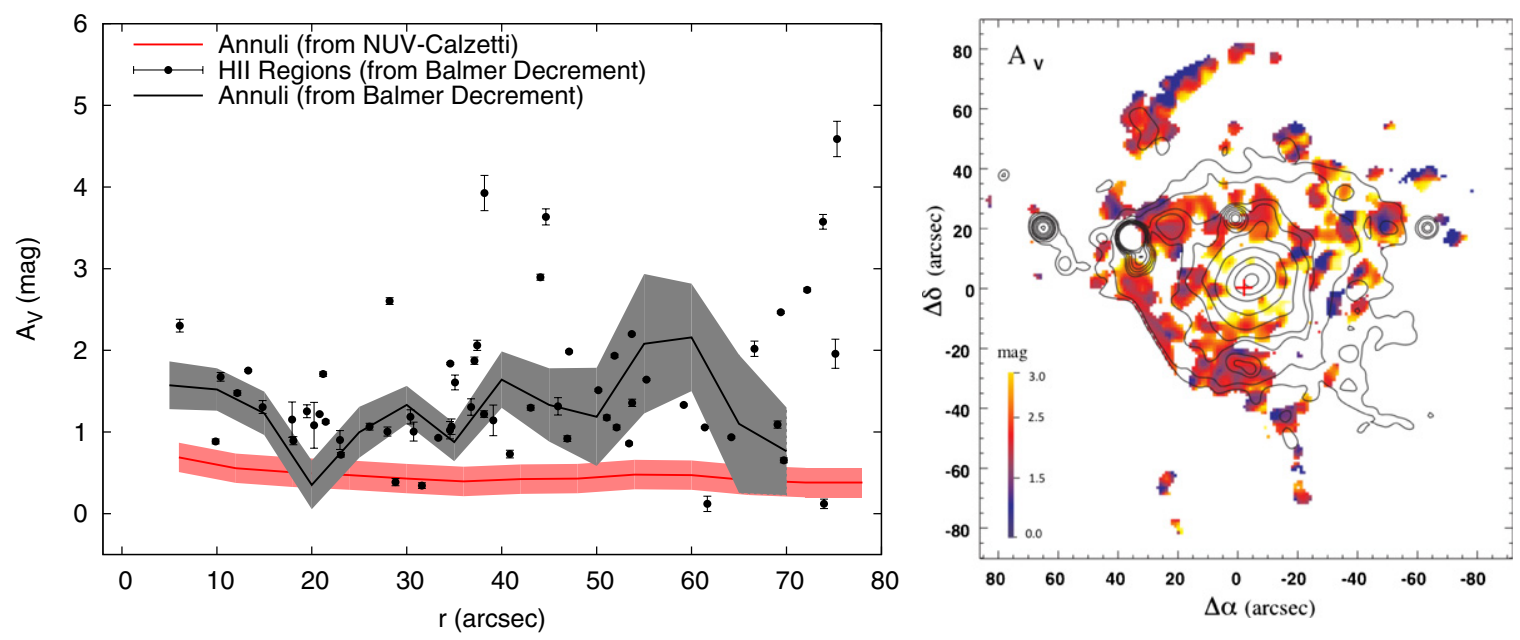

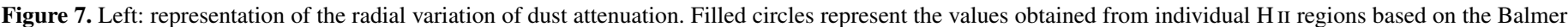

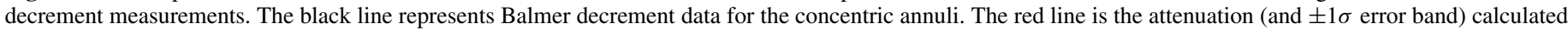

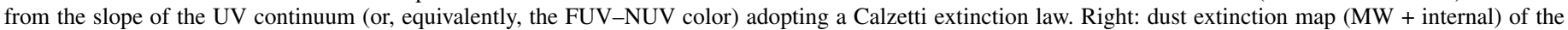
ionized gas in NGC 5668 computed from the $\mathrm{H} \alpha / \mathrm{H} \beta$ ratio. The $\mathrm{H} \alpha$ continuum values are represented as isocontours. North is up and east is to the left.

(A color version of this figure is available in the online journal.)

from atomic theory. Under case $\mathrm{B}$, conditions with temperatures $\sim 10,000 \mathrm{~K}$ and electron densities $\sim 100 \mathrm{~cm}^{3}$, the theoretical $\mathrm{H} \alpha / \mathrm{H} \beta$ ratio should be close to 2.86 (Osterbrock \& Ferland 2006). Since extinction is more severe in $\mathrm{H} \beta$ than in $\mathrm{H} \alpha$ wavelengths, the net effect is to increase the observed Balmer line ratio. As we discussed above, the results are dependent on the dust model of choice. Here, we assume that the dust is located in a uniform screen between us and the gas and that the extinction law in the optical is similar to the Galactic one, as parameterized by Cardelli et al. (1989).

\subsubsection{Optical-continuum Attenuation}

The last method that we have used for the extinction is based on our IDL fitting program for the underlying stellar population (see Section 4.1). From the output values for each concentric annuli, we find a mean value of $A_{V} \sim 1.7$ mag. This value has a very large uncertainty due to the limited wavelength range of our spectroscopic observations, which leads with strong age-metallicity-extinction-SFH degeneracies (see Gil de Paz \& Madore 2002). The results are summarized in Figure 7 . In general, the ionized-gas dust attenuation has a mean value of $\sim 1.5 \mathrm{mag}$, which is in agreement with what is found in other spiral disks (Gil de Paz et al. 2007). We find that the gas attenuation is larger than the continuum attenuation by $\left\langle E(B-V)_{\text {continuum }}\right\rangle=0.36 \times\left\langle E(B-V)_{\text {ionized-gas }}\right\rangle$ when the Calzetti attenuation law is adopted; note that ionized-gas color excess (derived from the Balmer decrement) is corrected for a MW foreground reddening, $E(B-V)=0.037$. This is somewhat expected given that, as shown in Calzetti et al. (2000) and Stasińska \& Sodré (2001), in nearby spiral galaxies there is evidence that stars, gas, and dust are typically decoupled (see, e.g., Maíz-Apellániz et al. 1998), so the attenuation inferred from the $\mathrm{H} \alpha / \mathrm{H} \beta$ ratio is typically higher than that inferred from the spectral continuum of the same wavelength (e.g., Calzetti et al. 1994; Mayya \& Prabhu 1996; Calzetti 1997). Poggianti et al. (1999) and Poggianti \& Wu (2000) explain such results are due to selective-dust-extinction effects where a large fraction, but not all, of the dust in galaxies is associated with star formation regions, which absorb a significant fraction of the light emitted by the young stars.

\subsection{Electron Density}

The electron density, $N_{e}$, is one of the key physical parameters that characterize gaseous nebulae. Most of the density estimates found in the literature are based on measurements of a sensitive emission-line ratio. In the presence of internal variations of electron density, however, these single line ratio measurements may not be representative of all ionizing zones. The measurements are based on the fact that a relation exists between the collisional de-excitation of atoms and electron density (Osterbrock \& Ferland 2006). If an ion emits similar amounts of energy from different energy levels at nearby wavelengths, then the ratio of the emission-line intensities can be used to obtain an estimate of the electron density and there are two line ratios that can be used: [O II] $\lambda \lambda 3726,3729$ or [S II] $\lambda \lambda 6717,6731$. In our case, we make use of the $[\mathrm{S} \mathrm{II}] \lambda \lambda 6717,6731$ line ratio and the electron density of the region responsible for the [S II] emission can be determined as

$$
R\left(\left[\mathrm{~S}_{\mathrm{II}}\right]\right)=\frac{I([\mathrm{~S} \mathrm{II}] \lambda 6717)}{I\left(\left[\mathrm{~S} \mathrm{II}_{\mathrm{II}}\right] \lambda 6731\right)} \simeq 1.49\left(\frac{1+3.77 x}{1+12.8 x}\right),
$$

where $x$, the density parameter, is defined as $x=10^{-4} \cdot n_{e} \cdot t^{-1 / 2}$. Solving this equation for the density parameter, we can obtain $N_{e}$ by assuming $T_{e}=T_{[\mathrm{S} \text { II }} / 10^{4}$, where $T_{[\mathrm{S} \mathrm{II}]}$ is the electron temperature of the region responsible for the [S II] emission (McCall et al. 1985). Thus, in order to properly estimate this electron density, a previous knowledge of the electron temperature of the region responsible for the [S II] emission lines is required. Assuming the calibration of $T[\mathrm{~N}$ II] as a function of the $R_{23}$ line ratio (see Equation (2)) given by Thurston et al. (1996) and an average difference of $3000 \mathrm{~K}$ between $T$ [N II] and $T\left[\mathrm{~S}_{\mathrm{II}}\right]$ (with the former being lower; Garnett 1992), we estimated $T_{[\mathrm{S}}$ iI] in the equation above. Note that although this estimate for the temperature is not very precise (as it does not rely on the use of temperature-sensitive line ratios), it is accurate enough for correcting the densities obtained from the $\left[\mathrm{S}_{\mathrm{II}}\right] \lambda \lambda 6717,6731$ line ratio for temperature effects. The large uncertainties associated with the determination of $T[\mathrm{~S}$ II] prevent us from extracting further conclusions from the density measurement derived. Despite that, we find a mean value for $N_{e}$ 
Table 6

Abundance Gradient Fits

\begin{tabular}{|c|c|c|c|c|c|c|}
\hline \multirow[t]{2}{*}{ Fits Type } & \multirow[t]{2}{*}{$r_{\text {BREAK }}$} & \multirow[t]{2}{*}{ Segment } & \multirow[t]{2}{*}{$12+\log (\mathrm{O} / \mathrm{H})$ at $r=0$} & \multicolumn{3}{|c|}{ Gradient } \\
\hline & & & & $\left(\operatorname{dex} /{ }^{\prime \prime}\right)$ & $\left(\operatorname{dex~} \mathrm{kpc}^{-1}\right)$ & $\left(\mathrm{dex} / R_{25}\right)$ \\
\hline Single slope & $\ldots$ & $\ldots$ & $8.640 \pm 0.047$ & $-0.0042 \pm 0.0009$ & $0.0346 \pm 0.0074$ & $0.416 \pm 0.089$ \\
\hline \multirow{2}{*}{ Double slope unweighted } & $40^{\prime \prime}$ & Inner & $8.876 \pm 0.056$ & $-0.013 \pm 0.002$ & $0.1073 \pm 0.0165$ & $1.29 \pm 0.20$ \\
\hline & & Outer & $8.269 \pm 0.012$ & $0.002 \pm 0.002$ & $0.002 \pm 0.019$ & $0.198 \pm 0.198$ \\
\hline \multirow[t]{2}{*}{ Double slope weighted } & $36^{\prime \prime}$ & Inner & $8.949 \pm 0.053$ & $-0.017 \pm 0.002$ & $0.140 \pm 0.016$ & $1.683 \pm 0.198$ \\
\hline & & Outer & $8.269 \pm 0.012$ & $0.002 \pm 0.002$ & $0.002 \pm 0.019$ & $0.198 \pm 0.198$ \\
\hline
\end{tabular}

of $190 \mathrm{~cm}^{-3}$ for the $\mathrm{H}$ II regions in agreement with the typical value densities in $\mathrm{H}$ II regions $\left(N_{e} \sim 10^{2} \mathrm{~cm}^{-3}\right.$; Osterbrock \& Ferland 2006).

\subsection{Chemical Abundances}

$\mathrm{H}$ II regions identify the sites of recent massive star formations in galaxies. The rapid evolution of these stars, ending in $\mathrm{SNe}$ explosions, and the subsequent recycling of nucleosynthesis products into the ISM, make $\mathrm{H}$ II regions essential probes for the present-day chemical composition of star-forming galaxies across the universe. The study of nebular abundances is therefore crucial for understanding the chemical evolution of galaxies. Obtaining a direct measurement of chemical abundances in H II regions requires a good estimate of the electron temperature. Unfortunately, this implies detecting very faint auroral lines such as [O III] $\lambda 4363$ or [N $\mathrm{NI}] \lambda 5755$, that are very faint at the abundance levels of most spiral disks. Thus, we must use instead calibrations based on the predictions of photoionization models or on empirical measurements for strong lines. Among the various strong-line methods, the $R_{23}$ indicator originally proposed by Pagel et al. (1979) stands out as arguably the most popular:

$$
R_{23}=\frac{f\left(\left[\mathrm{O}_{\mathrm{III}}\right] \lambda \lambda 4959,5007\right)+f([\mathrm{O} \mathrm{II}] \lambda \lambda 3726,3729)}{f(\mathrm{H} \beta)} .
$$

Like all strong-line diagnostics, $R_{23}$ as an abundance indicator has a statistical value, based on the fact that the hardness of the ionizing radiation correlates with metallicity. Numerous calibrations of $R_{23}$ in terms of the nebular chemical composition can be found in the literature. We choose as the best set of values for the oxygen abundance those given by the Kewley \& Dopita (2002) recipe, which basically adopts a calibration of the $[\mathrm{N} \mathrm{II}] /\left[\mathrm{O}_{\mathrm{II}}\right]$ ratio for oxygen abundances above half-solar and their own calibration of $R_{23}$ for abundances below that value. ${ }^{19}$ The $R_{23}$ abundance diagnostic depends strongly on the ionization parameter, for this reason we also calculated it following the indications given in Kewley \& Dopita (2002). These authors used a combination of stellar population synthesis and photoionization models to develop a set of ionization parameter and abundance diagnostics based only on the use of strong optical emission lines. These techniques are applicable to all metallicities. In particular, for metallicities above half-solar, the ratio $[\mathrm{N}$ II $] /[\mathrm{O} \mathrm{II}]$ provides a very reliable diagnostic since it is an independent ionization parameter and does not have a local maximum. This ratio has not been used historically because of concerns about reddening corrections. However, the use of classical reddening curves is sufficient to allow this [N II $] /[\mathrm{O}$ II] diagnostic to be used with confidence as a reliable abundance indicator. Note that the calibration of the $\left[\mathrm{N}_{\mathrm{II}}\right] /[\mathrm{O}$ II $]$ line ratio

\footnotetext{
19 We used the value of $12+\log (\mathrm{O} / \mathrm{H})_{\odot}=8.93$ as in Kewley \& Dopita (2002).
}

as a diagnostic of $12+\log (\mathrm{O} / \mathrm{H})$ relies on the dependence of the $(\mathrm{N} / \mathrm{O})$ abundance ratio on the oxygen abundance. Thus, no attempt has been made to derive the nitrogen abundance in this work.

The iterative method used as part of this recipe allows both these parameters to be obtained without the need of using temperature-sensitive line ratios involving very faint emission lines that are particularly elusive in the case of spiral disks. One drawback of using $R_{23}$ (and many other emission-line abundance diagnostics; see Pérez-Montero \& Díaz 2005) is that it depends also on the ionization parameter $q$ defined here as $q=S_{\mathrm{H} 0} / n$, where $S_{\mathrm{H} 0}$ is the ionizing photon flux through a unit area and $n$ is the local number density of hydrogen atoms. Some calibrations have attempted to take this into account (e.g., McGaugh 1991), but others do not (e.g., Zaritsky et al. 1994). Another difficulty in the use of $R_{23}$ and many other emissionline abundance diagnostics is that they are double valued in terms of the abundance $(12+\log (\mathrm{O} / \mathrm{H}))$. Thus, one of the main difficulties in its adoption is related to the necessity of locating which of two branches (upper and lower) a given $\mathrm{H}$ II region belongs to, since $R_{23}$ is degenerate (Bresolin et al. 2009a). This is because at a low abundance the intensity of the forbidden lines scales roughly with the chemical abundance while at high abundance the nebular cooling is dominated by the infrared fine structure lines and the electron temperature becomes too low to collisionally excite the optical forbidden lines. When only double-valued diagnostics are available, an iterative approach that explicitly solves for the ionization parameter, as the one used here that is based on the Kewley and Dopita logical flow diagram, helps to resolve the abundance ambiguities. In Tables 6 and 7, we present, the results respectively, derived for the abundance gradient Fits and the values obtained for both $\mathrm{H}$ II regions and concentric annuli. The metallicity values vary between $12+\log (\mathrm{O} / \mathrm{H})=8.15$ and $12+\log (\mathrm{O} / \mathrm{H})=8.7$ (i.e., from approximately $1 / 3$ the solar oxygen abundance to nearly the solar value). The lack of some regions in this table is due to the poor signal to noise or simply non-detection of $\mathrm{H} \beta$ emission (12 regions).

Simulations of the line flux errors were carried out in order to derive the errors in the oxygen abundances. These simulations assumed a Gaussian probability distribution for each line flux and that these were not correlated between the different lines. The resulting errors, which are shown in Table 7, could then be considered as upper limits to the actual line flux errors (where a contribution of correlated errors is expected).

In order to quantify the abundance gradient, we have carried out three linear regressions to the data points, the first one is a fit to all points weighted by their errors $\left(\chi_{\text {red }}^{2}=1.45\right)$; the gradient has a value of $-0.035 \mathrm{dex} \mathrm{kpc}^{-1}\left(-0.0042 \mathrm{dex} \operatorname{arcsec}^{-1}\right)$. The second and the third fits are double fits to the data with a free parameter, the radius of break, but one is weighted by the errors (black solid line) and the other is unweighted (black dashed line; yield both $\chi_{\text {red }}^{2}=0.54$ ). 
Table 7

Ionized-gas Diagnostics in the H II Regions and Concentric Annuli

\begin{tabular}{|c|c|c|c|c|c|}
\hline $\begin{array}{l}\text { ID } \\
(1)\end{array}$ & $\begin{array}{c}12+\log (\mathrm{O} / \mathrm{H}) \\
(2)\end{array}$ & $\begin{array}{c}q \\
(3)\end{array}$ & $\begin{array}{l}N_{e} \\
(4)\end{array}$ & $\begin{array}{l}T_{e} \\
(5)\end{array}$ & $\begin{array}{l}\text { Offsets } \\
\text { (6) }\end{array}$ \\
\hline \multicolumn{6}{|c|}{ Rings } \\
\hline 1 & $\ldots$ & $\ldots$ & 1137 & 53359 & $(-3.6,2.5)$ \\
\hline 2 & $\ldots$ & $\ldots$ & 206 & 11036 & $\ldots$ \\
\hline 3 & $8.69 \pm 0.11$ & $8.9 \mathrm{E}+06$ & 122 & 9822 & $\ldots$ \\
\hline 4 & $8.60 \pm 0.16$ & $9.5 \mathrm{E}+06$ & 186 & 9685 & $\ldots$ \\
\hline 5 & $8.63 \pm 0.32$ & $2.2 \mathrm{E}+07$ & 138 & 8362 & $\ldots$ \\
\hline 6 & $8.61 \pm 0.18$ & $1.1 \mathrm{E}+07$ & 169 & 9721 & $\ldots$ \\
\hline 7 & $8.50 \pm 0.13$ & $1.5 \mathrm{E}+07$ & 154 & 10184 & $\ldots$ \\
\hline 8 & $8.29 \pm 0.18$ & $1.7 \mathrm{E}+07$ & 106 & 9215 & $\ldots$ \\
\hline 9 & $8.50 \pm 0.12$ & $1.6 \mathrm{E}+07$ & 84 & 11308 & $\ldots$ \\
\hline 10 & $8.21 \pm 0.63$ & $1.7 \mathrm{E}+07$ & 27 & 11204 & $\ldots$ \\
\hline 11 & $\ldots$ & $\ldots$ & 256 & 10124 & $\ldots$ \\
\hline 12 & $\ldots$ & $\ldots$ & 248 & 15785 & $\ldots$ \\
\hline 13 & $\ldots$ & $\ldots$ & 287 & 16225 & $\ldots$ \\
\hline 14 & $\ldots$ & $\ldots$ & 242 & 11186 & $\ldots$ \\
\hline 15 & $\ldots$ & $\ldots$ & 56 & 10296 & $\ldots$ \\
\hline \multicolumn{6}{|c|}{ H II regions } \\
\hline 1 & $7.96 \pm 0.74$ & $2.5 \mathrm{E}+07$ & 123 & 9549 & $(-71.9,17.1)$ \\
\hline 2 & $7.43 \pm 1.08$ & $2.0 \mathrm{E}+07$ & $\ldots$ & 9229 & $(-62.8,20.3)$ \\
\hline 3 & $8.34 \pm 0.12$ & $1.7 \mathrm{E}+07$ & $\ldots$ & 9984 & $(-31.5,35.8)$ \\
\hline 4 & $8.36 \pm 0.07$ & $1.1 \mathrm{E}+07$ & $\ldots$ & 9247 & $(-52.1,25.9)$ \\
\hline 5 & $8.22 \pm 0.11$ & $1.6 \mathrm{E}+07$ & $\ldots$ & 9211 & $(-51.7,20.1)$ \\
\hline 6 & $\ldots$ & $\ldots$ & 272 & $\ldots$ & $(-50.7,53.6)$ \\
\hline 7 & $7.00 \pm 1.20$ & $\ldots$ & $\ldots$ & 10372 & $(-45.7,38.5)$ \\
\hline 8 & $8.30 \pm 0.21$ & $\ldots$ & 405 & 9229 & $(-40.4,-0.5)$ \\
\hline 9 & $8.34 \pm 0.10$ & $1.7 \mathrm{E}+07$ & $\ldots$ & 9935 & $(-39.9,-7.1)$ \\
\hline 10 & $\ldots$ & $\ldots$ & 10 & 10084 & $(-38.2,18.9)$ \\
\hline 11 & $8.38 \pm 0.11$ & $1.7 \mathrm{E}+07$ & 68 & 11163 & $(-34.9,32.2)$ \\
\hline 12 & $8.33 \pm 0.11$ & $1.8 \mathrm{E}+07$ & 33 & 11368 & $(-34.5,38.2)$ \\
\hline 13 & $8.43 \pm 0.38$ & $1.3 \mathrm{E}+07$ & $\ldots$ & 11047 & $(-31.8,-3.9)$ \\
\hline 14 & $8.60 \pm 0.22$ & $1.5 \mathrm{E}+07$ & $\ldots$ & 9940 & $(-31.3,8.4)$ \\
\hline 15 & $8.39 \pm 0.70$ & $1.1 \mathrm{E}+08$ & 352 & 7959 & $(-30.9,-28.0)$ \\
\hline 16 & $8.17 \pm 0.08$ & $2.0 \mathrm{E}+07$ & 30 & 9175 & $(-26.2,29.5)$ \\
\hline 17 & $8.45 \pm 0.36$ & $1.8 \mathrm{E}+07$ & $\ldots$ & 15032 & $(-23.6,-36.8)$ \\
\hline 18 & $\ldots$ & $\ldots$ & $\ldots$ & 8371 & $(-21.6,51.2)$ \\
\hline 19 & $8.58 \pm 0.20$ & $1.3 \mathrm{E}+07$ & 11 & 9295 & $(-21.1,17.6)$ \\
\hline 20 & $8.55 \pm 0.97$ & $7.4 \mathrm{E}+07$ & 12 & 7556 & $(-21.0,-69.4)$ \\
\hline 21 & $8.62 \pm 0.09$ & $1.3 \mathrm{E}+07$ & 116 & 10033 & $(-20.7,8.8)$ \\
\hline 22 & $7.80 \pm 1.00$ & $1.1 \mathrm{E}+07$ & 142 & 10992 & $(-18.6,36.5)$ \\
\hline 23 & $8.18 \pm 0.08$ & $3.9 \mathrm{E}+07$ & 78 & 10008 & $(-15.9,-42.8)$ \\
\hline 24 & $8.48 \pm 0.12$ & $1.1 \mathrm{E}+07$ & 12 & 9657 & $(-14.8,47.1)$ \\
\hline 25 & $8.50 \pm 0.23$ & $2.8 \mathrm{E}+07$ & 37 & 10573 & $(-14.2,-58.0)$ \\
\hline 26 & $8.72 \pm 0.25$ & $1.0 \mathrm{E}+07$ & 42 & 8739 & $(-13.6,2.8)$ \\
\hline 27 & $\ldots$ & $\ldots$ & $\ldots$ & 19479 & $(-12.5,77.3)$ \\
\hline 28 & $8.70 \pm 0.15$ & $1.0 \mathrm{E}+07$ & 103 & 9507 & $(-8.9,-6.5)$ \\
\hline 29 & $8.61 \pm 0.26$ & $1.4 \mathrm{E}+07$ & 29 & 8659 & $(-9.2,21.9)$ \\
\hline 30 & $8.58 \pm 0.25$ & $1.4 \mathrm{E}+07$ & 194 & 8860 & $(-7.5,-15.0)$ \\
\hline 31 & $8.25 \pm 0.15$ & $1.7 \mathrm{E}+07$ & $\ldots$ & 9175 & $(-5.7,-36.6)$ \\
\hline 32 & $8.50 \pm 0.23$ & $\ldots$ & $\ldots$ & 8699 & $(-3.7,-27.9)$ \\
\hline 33 & $8.69 \pm 0.09$ & $1.2 \mathrm{E}+07$ & 73 & 8941 & $(-2.3,-12.2)$ \\
\hline 34 & $8.39 \pm 0.21$ & $1.5 \mathrm{E}+07$ & $\ldots$ & 11198 & $(0.8,55.9)$ \\
\hline 35 & $8.66 \pm 0.07$ & $1.2 \mathrm{E}+07$ & $\ldots$ & 7534 & $(1.3,23.3)$ \\
\hline 36 & $8.41 \pm 0.04$ & $1.6 \mathrm{E}+07$ & $\ldots$ & 11831 & $(1.3,77.6)$ \\
\hline 37 & $8.48 \pm 0.24$ & $2.1 \mathrm{E}+07$ & 116 & 8804 & $(1.4,-24.9)$ \\
\hline 38 & $8.64 \pm 0.17$ & $1.0 \mathrm{E}+07$ & 50 & 9067 & $(3.3,-15.6)$ \\
\hline 39 & $\ldots$ & $\ldots$ & $\ldots$ & 13102 & $(3.3,-69.3)$ \\
\hline 40 & $8.47 \pm 0.18$ & $1.3 \mathrm{E}+07$ & $\ldots$ & 13174 & $(3.8,46.5)$ \\
\hline 41 & $\ldots$ & $\ldots$ & 168 & 8506 & $(5.3,-31.0)$ \\
\hline 42 & $8.57 \pm 0.13$ & $1.1 \mathrm{E}+07$ & $\ldots$ & 10684 & $(4.8,35.9)$ \\
\hline 43 & $8.61 \pm 0.39$ & $1.2 \mathrm{E}+07$ & 54 & 7507 & $(6.6,20.5)$ \\
\hline 44 & $8.74 \pm 0.05$ & $9.1 \mathrm{E}+06$ & 242 & 9523 & $(7.9,-0.7)$ \\
\hline 45 & $\ldots$ & $\ldots$ & 149 & 11738 & $(7.8,8.9)$ \\
\hline 46 & $8.57 \pm 0.26$ & $9.1 \mathrm{E}+06$ & 126 & 8596 & $(11.4,-18.7)$ \\
\hline 47 & $8.51 \pm 0.24$ & $1.1 \mathrm{E}+07$ & 241 & 8870 & $(11.5,-25.1)$ \\
\hline
\end{tabular}

Table 7

(Continued)

\begin{tabular}{lccccc}
\hline \hline ID & $12+\log (\mathrm{O} / \mathrm{H})$ & $q$ & $N_{e}$ & \multicolumn{1}{c}{$T_{e}$} & $\begin{array}{c}\text { Offsets } \\
(1)\end{array}$ \\
$(2)$ & $(3)$ & $(4)$ & $(5)$ & $(6)$ \\
\hline 48 & $8.63 \pm 0.12$ & $8.5 \mathrm{E}+06$ & 130 & 13086 & $(13.6,-9.6)$ \\
49 & $8.42 \pm 0.11$ & $2.8 \mathrm{E}+07$ & 170 & 10808 & $(14.8,68.9)$ \\
50 & $8.54 \pm 0.30$ & $1.4 \mathrm{E}+07$ & 201 & 8500 & $(19.0,-1.1)$ \\
51 & $8.47 \pm 0.14$ & $1.7 \mathrm{E}+07$ & 118 & 9599 & $(20.3,25.5)$ \\
52 & $8.53 \pm 0.27$ & $1.9 \mathrm{E}+07$ & 137 & 8655 & $(23.8,15.9)$ \\
53 & $8.31 \pm 0.10$ & $1.5 \mathrm{E}+07$ & 158 & 9683 & $(25.0,21.6)$ \\
54 & $8.43 \pm 0.08$ & $2.6 \mathrm{E}+07$ & $\ldots$ & 10588 & $(25.6,59.5)$ \\
55 & $8.44 \pm 0.08$ & $2.4 \mathrm{E}+07$ & 54 & 10645 & $(30.7,50.5)$ \\
56 & $\ldots$ & $\ldots$ & 50 & 6785 & $(33.3,8.9)$ \\
57 & $8.57 \pm 0.16$ & $8.1 \mathrm{E}+06$ & 259 & 23652 & $(34.3,-0.0)$ \\
58 & $8.34 \pm 0.21$ & $1.9 \mathrm{E}+07$ & 170 & 11032 & $(42.5,29.0)$ \\
59 & $8.34 \pm 0.37$ & $1.0 \mathrm{E}+07$ & 1318 & 12890 & $(45.2,13.2)$ \\
60 & $7.96 \pm 0.83$ & $1.1 \mathrm{E}+07$ & 335 & 10993 & $(48.3,2.9)$ \\
61 & $\ldots$ & $\ldots$ & 619 & 19051 & $(69.1,14.2)$ \\
62 & $\ldots$ & $\ldots$ & 60 & 12023 & $(-3.2,4.3)$ \\
\hline
\end{tabular}

Notes. (1) H II regions and annuli identification. (2) Oxygen abundances along with the corresponding errors. (3) Ionization parameter $q$ defined as $q=S_{\mathrm{H} 0} / n$. (4) Electron density in units of $\mathrm{cm}^{-3}$. (5) Electron temperature in Kelvin. (6) Offset coordinates of the $\mathrm{H}$ II regions in units of arcsec.

The Kewley \& Dopita recipe allows us to make use of the most optimal abundance indicator among [N II]/[O II], [N II] $/ \mathrm{H} \alpha$, and $R_{23}$. We find that the three abundance diagnostics (shown in the bottom panel of Figure 8 with different colors) are homogeneously mixed, as shown in Figure 8 . Thus, the $[\mathrm{N} \mathrm{II}] / \mathrm{H} \alpha$ and $R_{23}$-based abundance values are well mixed and can be derived at almost any galaxy radius, while the $[\mathrm{N} \mathrm{II}] /[\mathrm{O}$ II] indicator is lost in some $\mathrm{HII}$ regions, mostly at large radii. This is, as pointed out in Kewley \& Dopita (2002), because nitrogen is predominantly a primary nucleosynthesis element in the range $12+\log (\mathrm{O} / \mathrm{H}) \leqslant 8.6$ (see also Figure 3 in Kewley \& Dopita 2002) and the calibration of the (N/O) abundance ratio and the $[\mathrm{N}$ II $] /[\mathrm{O}$ II $]$ line ratio is not sensitive to the oxygen abundance under these circumstances.

The results are summarized in Table 6 and Figure 8 where we plot the three gradients as a function of the de-projected galactocentric radius. We find that inward $r \sim 36^{\prime \prime}(\sim 4.4 \mathrm{kpc})$ the $\mathrm{O} / \mathrm{H}$ ratio follows an exponential profile with a slope of $0.140 \pm 0.016\left(\mathrm{dex} \mathrm{kpc}^{-1}\right)$ and $12+\log (\mathrm{O} / \mathrm{H})_{r=0} \simeq 8.9$, similar to the normalized radial gradient found in other spiral disks. The outer abundance trend flattens out to an approximately constant value of $12+\log (\mathrm{O} / \mathrm{H})_{r=0} \simeq 8.27$ (with a slight gradient of $\left.0.002 \pm 0.019\left(\mathrm{dex} \mathrm{kpc}^{-1}\right)\right)$ and could even reverse (see Section 6 for a discussion on the possible causes for such flattening). The abundance gradient derived, $-0.035 \mathrm{dex} \mathrm{kpc}-1$ $\left(-0.0042 \mathrm{dex} \operatorname{arcsec}^{-1}\right)$, is somewhat shallower than the one for the MW (-0.08 dex kpc ${ }^{-1}$; Boissier \& Prantzos 1999 and references therein). A similar trend was found from the analysis of the spectra of the concentric annuli. The analysis of these spectra was limited to the innermost nine annuli, because beyond this ring both the continuum and line emission become too faint to derive reliable emission-line fluxes. In Figure 8, we plot the change in abundance for all annuli with a solid black line (bottom panel) where the uncertainties associated are represented by the gray shaded area. The blue dashed line represents the metallicity profile predicted by the best-fitting model of Boissier \& Prantzos (1999, 2000, hereafter BP2000 models). This profile has been shifted by 1 dex to match the metallicity scale derived from our spectroscopic data. Note that 


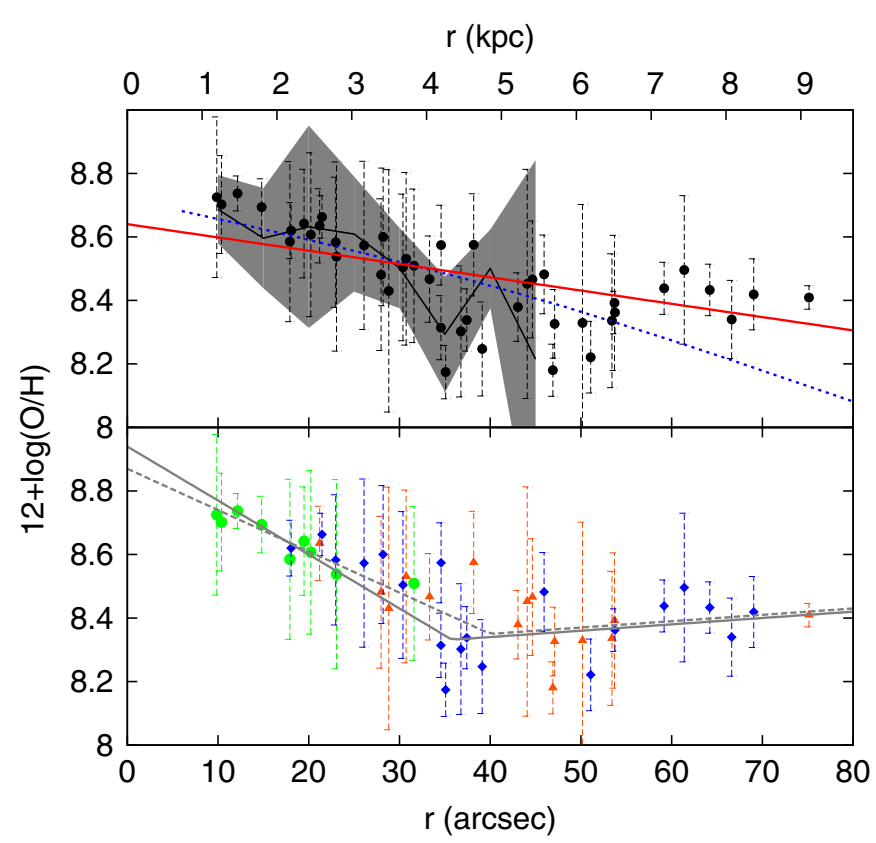

Figure 8. Radial abundance gradient in NGC 5668, where filled symbols correspond to individual HiI regions while the gray shaded area represents the values obtained for the concentric annuli. Top panel: the red line symbolizes the linear regression fit to the $\mathrm{H}$ II region data. We obtained a single gradient of $0.035 \pm 0.007 \mathrm{dex} \mathrm{kpc}^{-1}$. The blue dashed line represents the gradient obtained from the models of Boissier \& Prantzos (1999; BP2000), with an offset of $1 \mathrm{dex}$ in the metallicities plotted (see the text for details). Bottom panel: in this plot, we draw two types of fits: the gray line reproduces a double fit weighted by the errors; the gradient has a value of $0.140 \pm 0.016$ for inner part and $0.002 \pm 0.019$ dex for outer part. The gray dashed line reproduces the unweighted double fit that yields gradient values of $0.1073 \pm 0.0165$ (inner) and $0.002 \pm 0.019$ (outer). The color coding of the points represents the different line ratios used to calculate the metallicity for the central point of the ratios probability distribution (based on Kewley \& Dopita 2002 recipe): green points are calculated from $[\mathrm{N} \mathrm{II}] /[\mathrm{O} \mathrm{II}]$, red triangles represent the values obtained with [N II] $/ \mathrm{H} \alpha$, and blue diamonds show $R_{23}$-based values. Note that the presence of three uncertain points (regions 13, 17, and 59 have an error major of 0.3 ) has no influence on the fitting parameter values.

(A color version of this figure is available in the online journal.)

this kind of zero-point offsets in metallicity are not unexpected due to the significant uncertainties in the yields used in the disk evolution models (see discussion in Muñoz-Mateos et al. 2011); besides, even the empirical oxygen abundances can be subject to large systematic offsets (Moustakas et al. 2010). The relative changes in metal abundances and therefore their radial profiles, on the other hand, are much more robust to these unknowns.

\subsection{Galaxy Disk Modeling}

In order to gain further insight into the evolution of NGC 5668, we have fitted its multi-wavelength surface brightness profiles with the BP2000 models. These models describe the chemical and spectrophotometric evolution of spiral disks as a function of only two variables: the dimensionless spin parameter, $\lambda$, and the circular velocity in the flat regime of the rotation curve, $V_{\mathrm{C}}$. Within these models, galactic disks are simulated as a set of concentric rings that evolve independently from each other (for simplicity, radial mass or energy flows are not considered). The gas infall rate at each radius decreases exponentially with time, with a timescale that depends on both the total mass of the galaxy and the local mass surface density at that radius. Once in the disk, gas is transformed into stars following a Kennicutt-Schmidt law multiplied by a dynamical term, which accounts for the periodic passage of spiral density waves. The mass distribution of each new generation of stars follows a Kroupa (2001) initial mass function (IMF). The finite lifetimes of stars of different masses is taken into account; when they die, they inject metals into the ISM, thus affecting the metallicity of subsequent stars. The local metallicity at the time of formation is taken into account when determining the lifetimes, yields, evolutionary tracks, and spectra of each generation of stars. The model was first calibrated against several observables in the MW (see Boissier \& Prantzos 1999) and then extended to other galaxies with different values of $\lambda$ and $V_{\mathrm{C}}$ (BP2000), using several scaling laws derived from the $\Lambda$-Cold Dark Matter framework of galaxy formation (Mo et al. 1998).

For each pair of values of $\lambda$ and $V_{C}$, the model outputs radial profiles at different wavelengths, which can then be compared to the actual profiles of our Galaxy. In order to probe the spatial location of stars of different ages - and therefore better constrain the model predictions - we measured surface brightness profiles at all GALEX FUV and NUV bands, the ugriz bands from SDSS, and the 3.6 and $4.5 \mu \mathrm{m}$ Spitzer bands. The radial variation of internal extinction in the UV was estimated indirectly from the FUV-NUV color profiles, as explained in Section 5.1.1. The UV extinction was then extrapolated to the optical and nearIR bands, assuming a MW extinction curve, convolved with a sandwich model to account for the relative geometry of stars and dust (see Muñoz-Mateos et al. 2011 for details).

We compared these extinction-free profiles with the model predictions for a grid of values of $\lambda$ and $V_{C}$, and used an $\chi^{2}$ minimization algorithm to find the best-fitting values. The results of the fit are shown in Figure 9, and in Figure 10 we present the residuals. Each panel corresponds to a different wavelength. The gray profiles are only corrected for foreground MW extinction, whereas the black ones are corrected for internal extinction as well. The red and blue dashed lines bracket the region of the profile used for the fit. On the one hand, starlight inside $r \simeq 20^{\prime \prime}$ is dominated by emission coming from the bulge and the oval. On the other hand, beyond $r \simeq 125^{\prime \prime}$ the $\mathrm{S} / \mathrm{N}$ becomes very low, and contamination from background sources could be an issue. The model that best reproduces simultaneously all multi-wavelength profiles is shown with a red line. The shaded band encompasses all models whose $\chi^{2}$ is less than twice the $\chi^{2}$ of the best model $\left(\chi_{\min }^{2} \simeq 0.8\right) .{ }^{20}$ The resulting $\chi^{2}$ of the fitting model is obtained after a two-stagefitting procedure because, as one would expect, this model does not reproduce the very small-scale variations of the surface brightness profiles of our disk. So in a first run of the fitting procedure we assume that the total uncertainty for each point is due to the quadratic sum of the zero-point and photometric errors, plus an extra uncertainty in the model predictions of $10 \%$. These values represent the initial guess for the intrinsic error of the model and in this stage the $\chi^{2}$ is $\sim 5$. In the second step, we calculate the rms of the best-fitting model with respect to the galaxy profiles and we pass these error profiles to the code as initial uncertainties to start the second run. In this way the new reduced $\chi^{2}$ values are close to unity. In principle, when we

\footnotetext{
20 Note that the scatter of the data points around the model is not entirely due to noise, but also to real features of the galaxy that a smooth model cannot reproduce. This precludes applying the classical statistical formula to translate the $\chi^{2}$ distribution into confidence intervals. The criterion of using $\chi^{2} \leqslant 2 \chi_{\min }^{2}$ is just orientative, based on the results shown in Figure 9. Note also that we are not using the $\chi^{2}$ distributions in a strict statistical way, since many of the required mathematical conditions (Gaussianity of errors, etc.) are not met (see Muñoz-Mateos et al. 2011 for a more extensive discussion).
} 

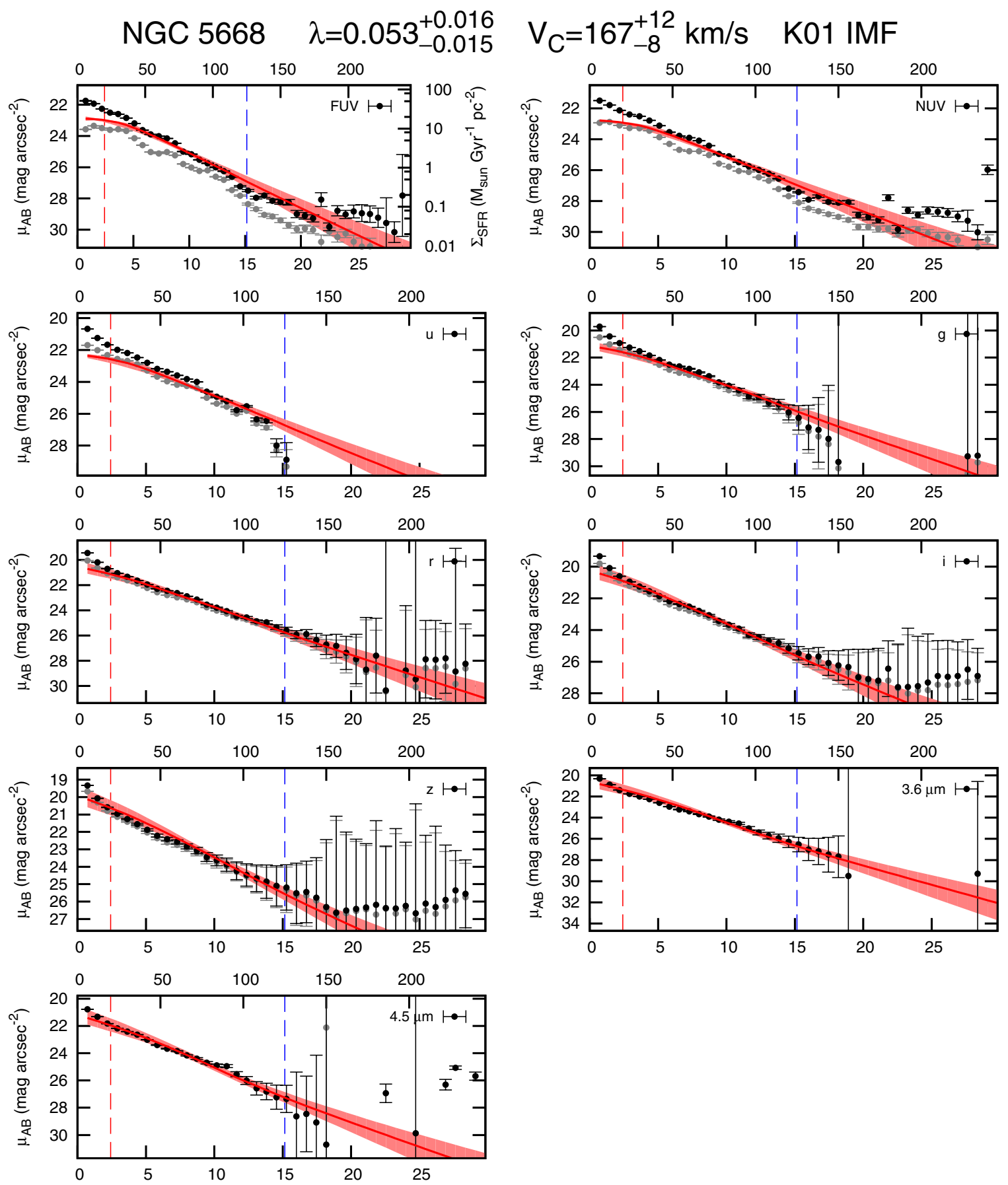

Figure 9. Best-fitting model of NGC 5668 using the Kroupa (2001) IMF. The gray profiles are only corrected for foreground Milky Way extinction, whereas the black ones are also corrected for internal attenuation. The latter profiles are the ones used to constrain the disk evolution models in the radial range spanned between the red and blue vertical lines (in order to exclude the bulge and the low $\mathrm{S} / \mathrm{N}$ outer parts). The best-fitting model is shown as a red solid line, and the red shaded band comprises all models with $\chi^{2} \leqslant 2 \chi_{\min }^{2}$. The radius along the semimajor axis is shown both in kpc (bottom $x$-axis) and arcseconds (top $x$-axis).

(A color version of this figure is available in the online journal.)

fit a set of data points with a given model, it is often implicitly assumed that deviations between the observed data points and the model are due to the measured uncertainties of the former. However, in practice one also has to account for the fact that the models themselves are not a perfect representation of nature and have their own "uncertainties." The model yields smooth profiles that, by construction, cannot reproduce the fine structure of the actual profiles. In order to account for this, we performed this fit for NGC 5668 in a two-stage fashion (see also Section 5 of Muñoz-Mateos et al. (2011) for a detailed discussion). When fitting the multi-wavelength profiles, we should not ignore the internal degeneracy in the determination of the $\lambda$ and $V_{\mathrm{C}}$ values as these are not completely independent parameters. For this reason in Figure 11, we show the two-dimensional $\chi^{2}$ distribution obtained for NGC 5668 in the case of fitting all bands simultaneously and in the case of each band separately. The corresponding best-fitting values are $\lambda=0.053_{-0.015}^{+0.016}$ and $V_{\mathrm{C}}=167_{-8}^{+12} \mathrm{~km} \mathrm{~s}^{-1}$. Where the errors are those expected when one is interested in deriving each of the two quantities separately. 
NGC $5668 \lambda=0.053_{-0.015}^{+0.016}$
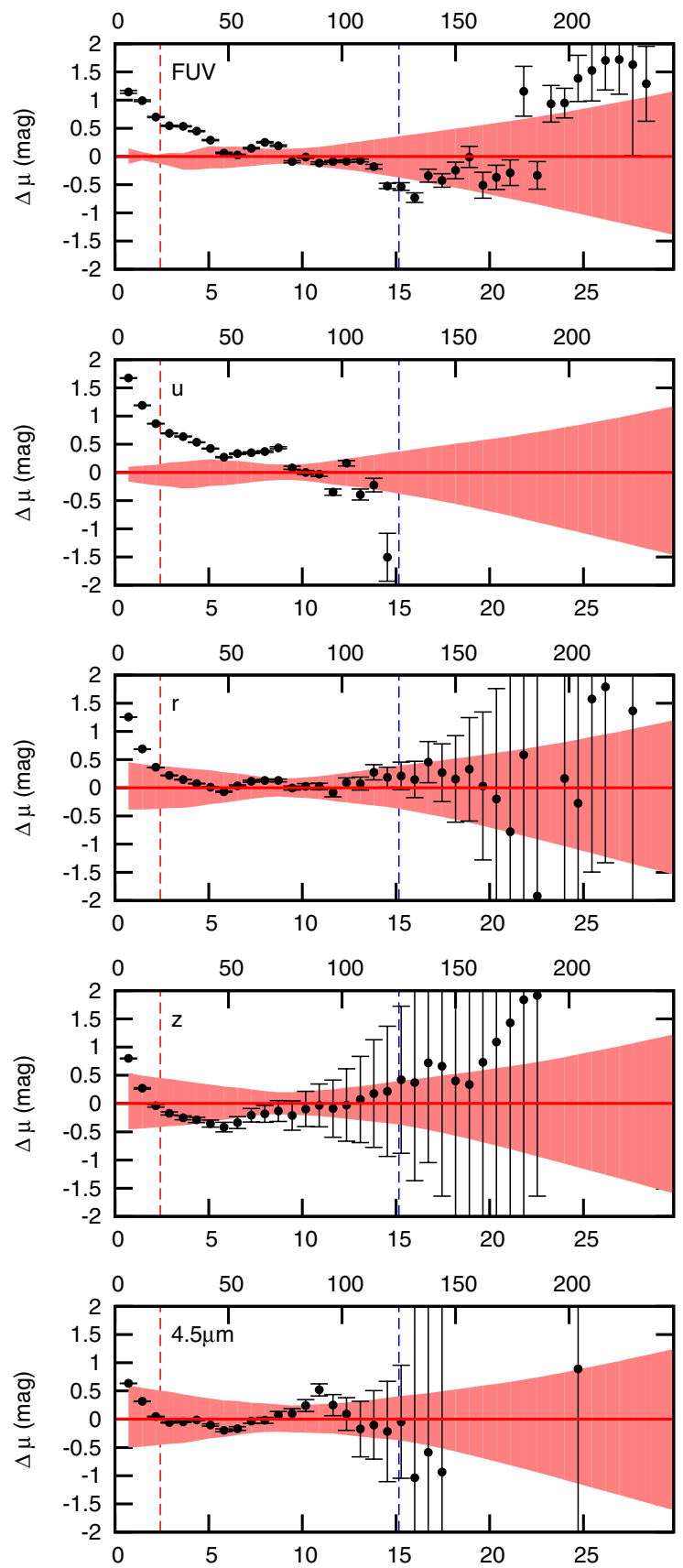

$\mathrm{V}_{\mathrm{C}}=167_{-8}^{+12} \mathrm{~km} / \mathrm{s} \quad \mathrm{K} 01 \mathrm{IMF}$
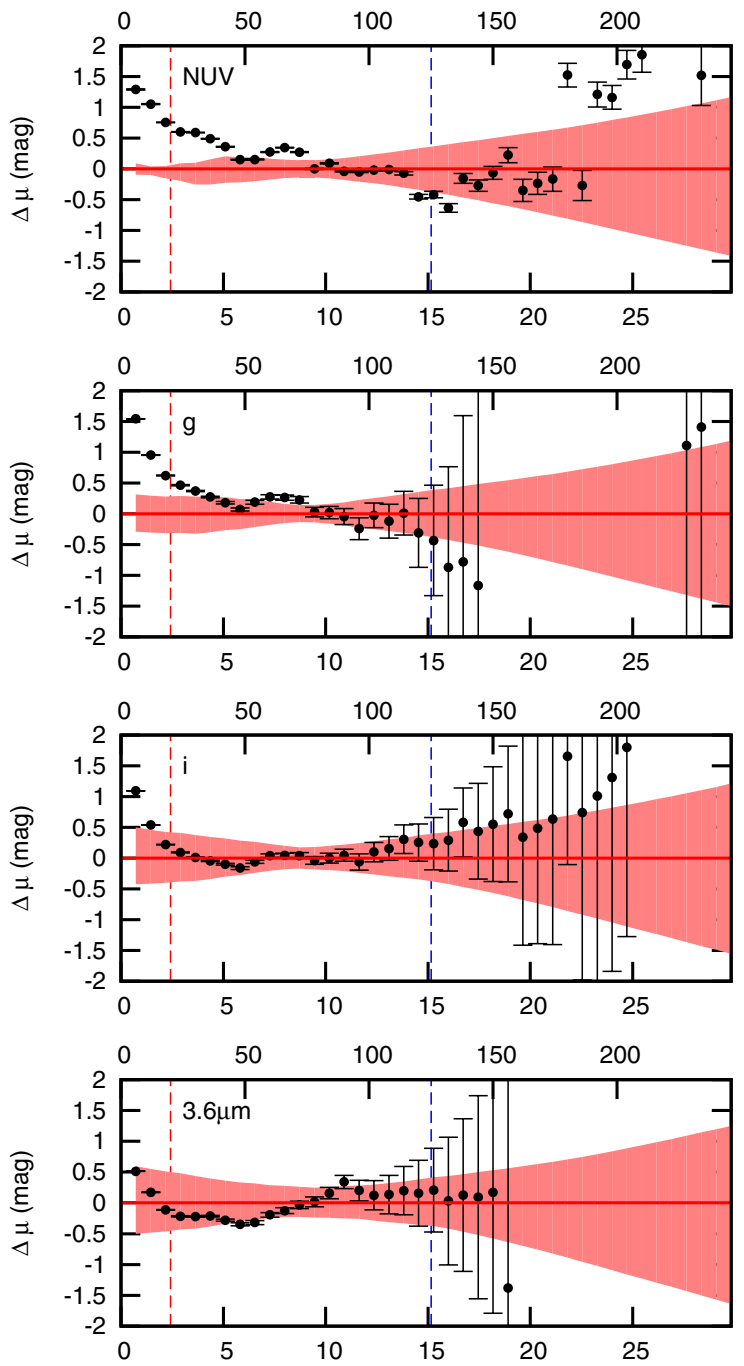

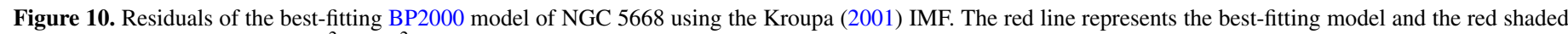

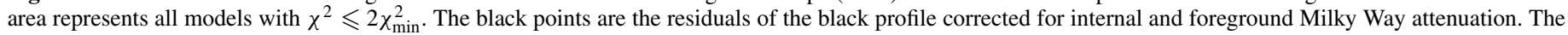
radius along the semimajor axis is shown both in kpc (bottom $x$-axis) and arcseconds (top $x$-axis).

(A color version of this figure is available in the online journal.)

In general, all bands are successfully reproduced by the BP2000 models, but the fit is not equally good at all wavelengths. For example, we can appreciate in the top panels of Figure 10 that in the cases of FUV, NUV, and $u$-band data the quality of the fit is not so good because these bands are more sensitive to the recent variations in the $\mathrm{SFH}$ and on the recipe used to calculate the extinction (or even limitations intrinsic to the models in order to reproduce the UV part of the spectrum at certain metallicities, as explained in Figure 9 of Muñoz-Mateos et al. 2011). The deviations in UV bands from the BP2000 models are compatible with the typical range of errors, as pointed out in Muñoz-Mateos et al. (2011).

While the BP2000 models provide a good fit to the overall shape of the surface brightness profiles of NGC 5668 (see Figure 9), the analysis of the deviations from these otherwise idealized models could give important clues on the details of the SFH of this galaxy (e.g., gas and/or stellar radial transfers). In this regard, Figure 12 shows that while the best-fitting model predicts a systematic bluing in the colors toward the outer parts of the disks, the measured colors flatten or even get redder 


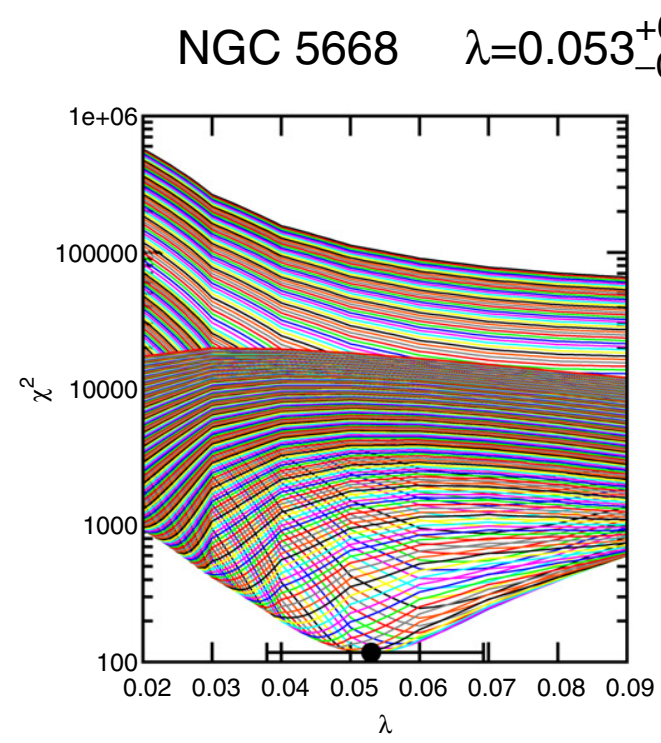
$-0.016$

$\mathrm{V}_{\mathrm{C}}=167_{-8}^{+12} \mathrm{~km} / \mathrm{s} \quad \mathrm{K} 01 \mathrm{IMF}$
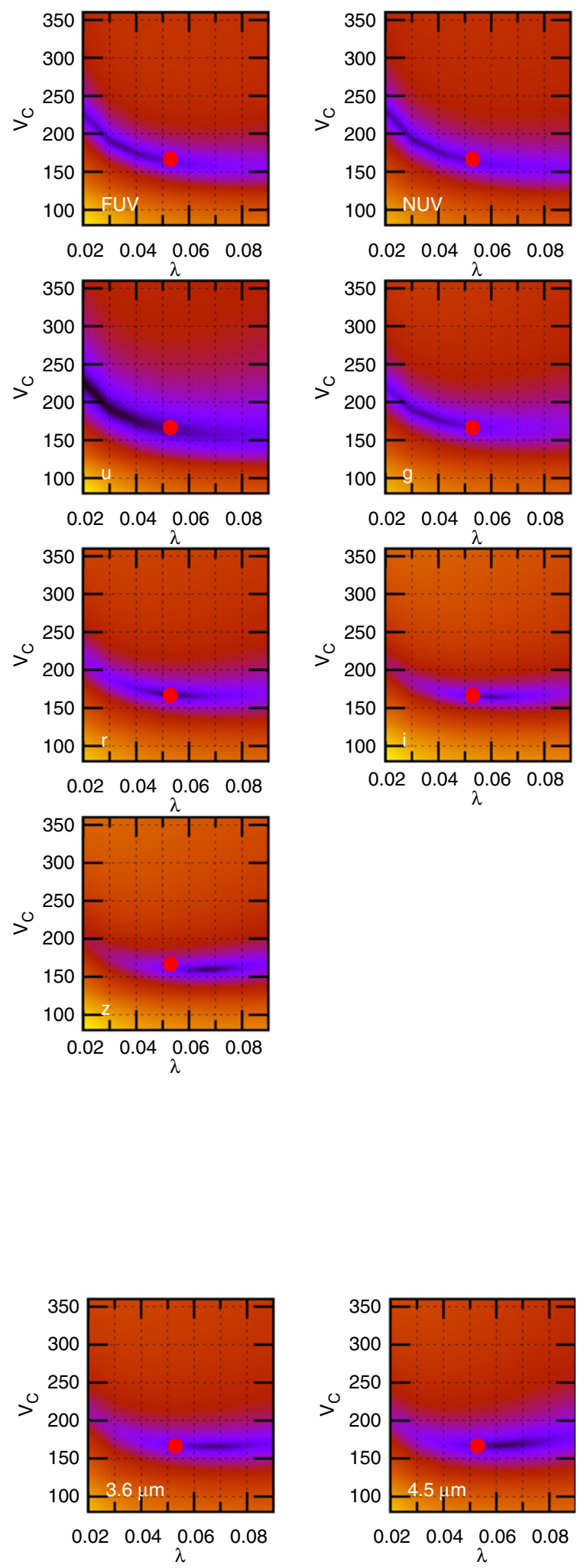

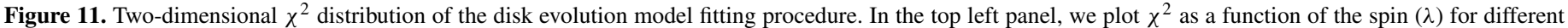

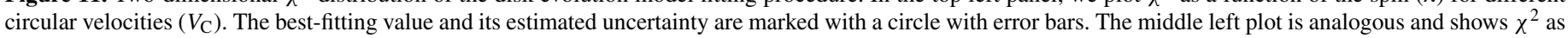

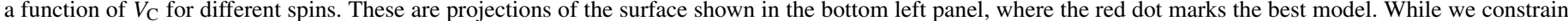

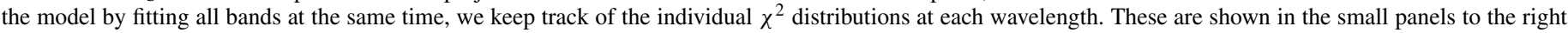

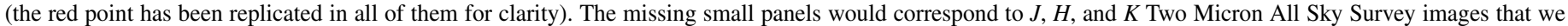
decided to exclude from this analysis as they are not deep enough to provide additional information. For more details, see Muñoz-Mateos et al. (2011).

(A color version of this figure is available in the online journal.) 


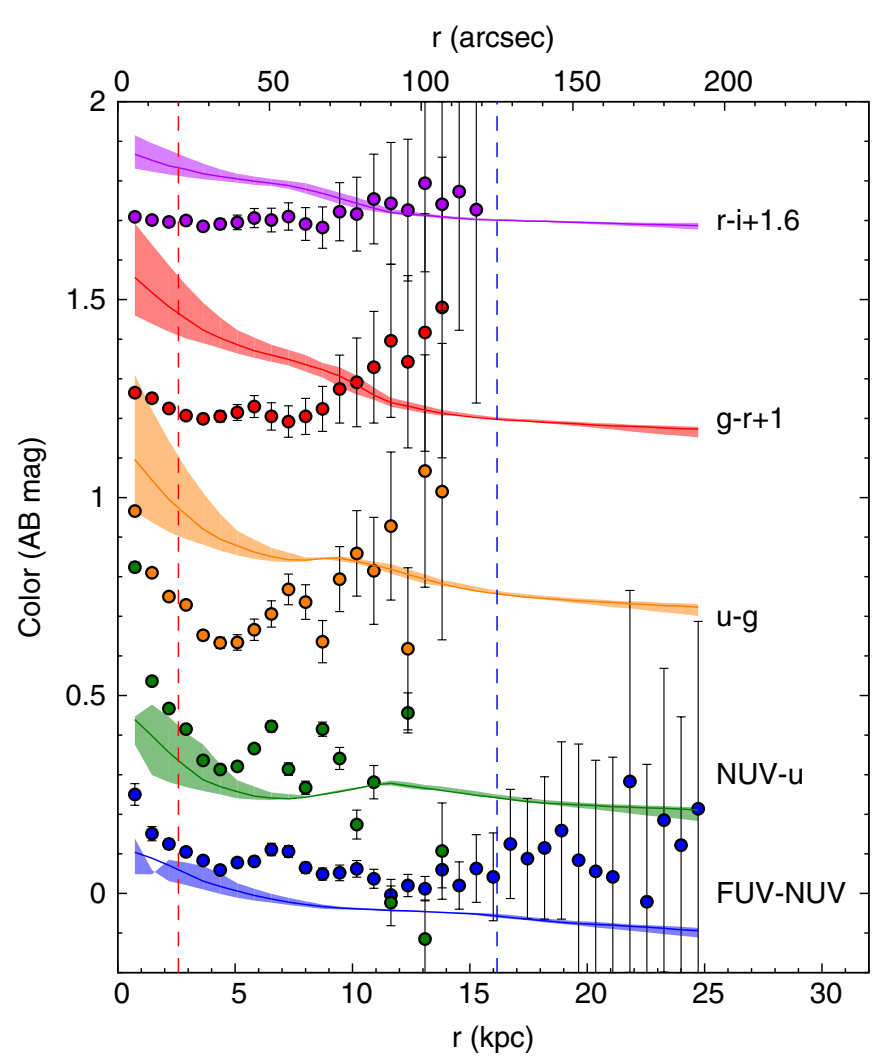

Figure 12. Observed color profiles of NGC 5668 (points) corrected for internal extinction as in the black points of Figure 9. These observed colors are compared with the ones predicted by the best-fitting model to light profiles (lines and bands). The red and blue vertical dashed lines bracket the spatial range used during the fit. Note that since the fit is performed simultaneously at all radii and all wavelengths from the FUV to $4.5 \mu \mathrm{m}$, departures up to a few tenths of a magnitude are expected for a particular color and radius.

(A color version of this figure is available in the online journal.)

beyond $\sim 30^{\prime \prime}-40^{\prime \prime}$. This radius interestingly coincides with the position where the slope of the metallicity gradient changes sign. We also find small offsets $(\leqslant 0.2 \mathrm{mag})$ between the observed and predicted colors. This is partially due to uncertainties in the model predictions for some of these colors.

The model has to reproduce at the same time the shape of the observed surface brightness profiles in all bands simultaneously and in addition it has to reproduce the "average" level of surface brightness. This kind of fitting introduces some limits in the range of the model parameters in a way that the colors of these models are located in a relatively narrow range as a consequence of the high degeneration within those model (surface brightness) profiles that match the data at all wavelengths simultaneously. ${ }^{21}$

Small variations of a few tenths of a magnitude will not have a significant impact on the overall shape of the surface brightness profiles, but will reveal themselves more clearly in the color profiles (see Muñoz-Mateos et al. 2011). On the other hand, the SFHs of the different annuli in the model are tied to one another (due to the analytical way in which we implement radial changes) but the actual SFH within the galaxy will exhibit more complex radial variations from one ring to the next.

\footnotetext{
21 One could decide to fit only the color profiles instead. However, that would lead to solutions that might not fit the overall shape of the surface brightness profiles and that would be very sensitive to uncertainties in the model predictions regarding specific colors (associated with limitations in the yields, stellar libraries at non-solar abundances, and uncertainties in the luminosity at near-infrared and UV wavelengths; see Maraston et al. 2006 and Muñoz-Mateos et al. 2011, respectively).
}

\section{DISCUSSION}

The analysis presented above shows that most of the properties of the disk of NGC 5668 can be well reproduced by the chemical and spectrophotometric BP2000 models. This is true both for the global metallicity gradient as well as for the shape of the galaxy surface brightness profiles. This is also true for the rotation curve (see Figure 13). However, there are observables (chemical abundances and colors) and, especially, radial intervals among them that are not so well fitted by these simple models. The objective must then be to establish the mechanism(s) that drive the departures of these observables and the associated star formation and chemical histories in NGC 5668 from those predicted by the inside-out scenario of the disk formation on which the BP2000 models are based on. In particular, we ought to explain the bimodality (Chiappini et al. 2001) of the abundance gradient (and color profiles) measured in NGC 5668: flatter in the outermost regions and steeper in the inner ones.

\subsection{Bimodal Distribution Mechanisms}

The re-distribution of stars, gas, and dust in galaxies can have important consequences in the metallicity distribution and, in particular, in deviations of the chemical and photometric properties of disks with respect to the predictions of static disk evolution models. In this regard, radial changes (up- or downbending) in the slope of the abundance gradient of nearby spiral galaxies (Vílchez \& Esteban 1996) are detected and are often related to gaseous and stellar radial mixing processes (Spitzer \& Schwarzschild 1953; Barbanis \& Woltjer 1967; Shaver et al. 1983; Fuchs 2001; Sellwood \& Binney 2002; Roškar et al. 2008; Haywood 2008; Bresolin et al. 2009b; Vlajić et al. 2009).

The existence of this kind of bimodal gradients are often proposed to have been induced by the presence of a bar-like potential (Díaz et al. 1990; Vila-Costas \& Edmunds 1992; Edmunds \& Roy 1993; Zaritsky et al. 1994). Indeed, in the case of strong-barred galaxies a change from a shallow to a steep metallicity profile is commonly observed and explained in the context of radial mixing of the gas induced by the bar (Martin \& Roy 1995; Roy \& Walsh 1997). On the other hand, bars can also lead to a steepening of the metallicity profile toward the inner disk if the gas transfer results in significant star formation in situ (Friedli et al. 1994; Martin \& Roy 1995; Roy et al. 1996; Roy \& Walsh 1997).

This latter behavior has been found in dynamical simulations of the formation of bars (Friedli et al. 1994; Friedli \& Benz 1995). In these simulations, the presence of a steep-shallow break in the metallicity profile is the result of an intense chemical enrichment by star formation in the bar combined with the dilution effect of the outward flow beyond the break. According to these models the presence of such a break indicates that the bar has recently formed, i.e., in the last Gyr. Observationally, the metallicity distribution of a number of galaxies hosting young bars has been successfully reproduced by this or a similar scenario (Roy \& Walsh 1997; Considère et al. 2000).

However, other mechanisms have been also proposed to explain bimodal metallicity distributions. Thus, in the context of the spiral density wave theory, star formation is expected to be proportional to $\Omega-\Omega_{p}$ (Oort \& Peixóto 1974). In Jensen et al. (1976), this prediction was successfully tested against the metallicity gradients measured in a number of grand-design spirals. Should this scenario be valid, one would expect to find a minimum in the chemical abundance at the position of the 

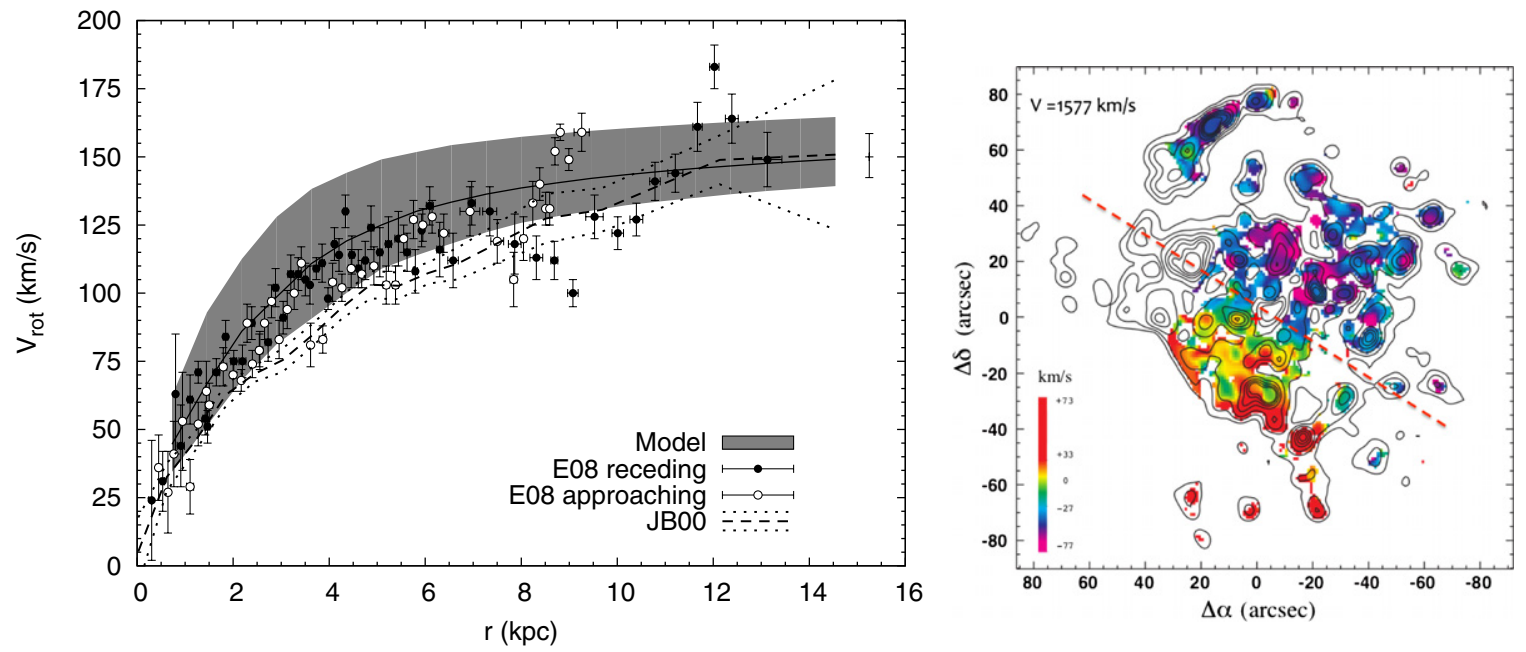

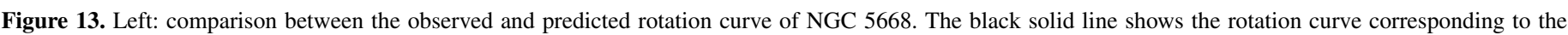

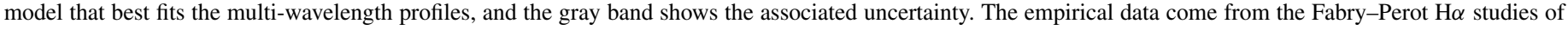

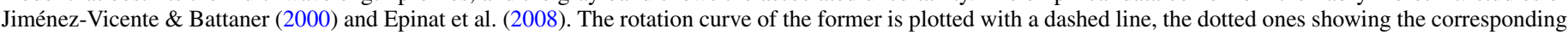

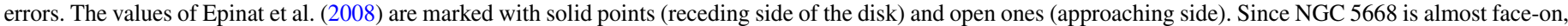

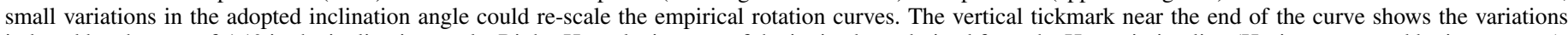

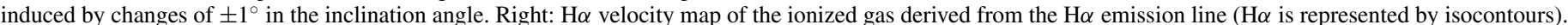
North is up and east is to the left. One pointing was masked out because the derived velocity values were not reliable.

(A color version of this figure is available in the online journal.)

corotation radius (see also the recent works by Acharova et al. 2005; Scarano 2010), especially in the case of galaxies where the spiral structure is long-lived and quasi-stationary (McCall 1986; Acharova et al. 2005). This is to the fact that $\Omega-\Omega_{p}$ is null (by definition) at corotation and, therefore, star formation should be less efficient resulting in a lesser degree of metal enrichment. In this scenario, a maximum in the colors should also be observed at the approximate position of the corotation radius as the optical-near-infrared colors would be dominated by those of the underlying stellar population since the current day SFR is expected to be low.

Finally, a flattening of the outer color and metallicity profiles is believed to be also caused by the increasing contribution of old migrated stars to the stellar content of disks (Zaritsky et al. 1994; Binney 2001; Sellwood \& Binney 2002; Roškar et al. 2008; more recently, Minchev et al. 2011; Roškar et al. 2011). In this case, as we move further out into the outer disk we find progressively older stars, which have been migrating for a longer period of time, leading to a positive age and color gradient in these regions. With regard to the metal abundance and according to the simulations of Roškar et al. (2008), radial migration leads to the mixing of the old stellar populations, which results in flatter gradients at early times and in the very outer regions of the present-day disks as these regions are primarily populated by migrated stars. In the few observational and theoretical studies on the luminosity-weighted age profiles of the disks, a similar flattening or even an up-bending is found (Bakos et al. 2008; Sánchez-Blázquez et al. 2009, 2012; Yoachim et al. 2010). While some authors favor stellar migration as the main driver of the shaping of these age profiles (Yoachim et al. 2010), some others indicate that this might be due to a decrease in the star formation in the external parts of the disk with time caused by a reduction of the volume density of the gas in these regions (Sánchez-Blázquez et al. 2009).

The different scenarios proposed above to explain a steepshallow break in the metallicity gradient of galaxies, namely, a young bar, reduced star formation at the corotation radius, stellar migration, or evolution of the star formation threshold with redshift have different imprints not only on the specific shape of the metallicity gradient but also on other properties such as the colors. In the case of NGC 5668, the change in the shape of the metallicity gradient takes place at a radius of $\sim 36^{\prime \prime}(4.4 \mathrm{kpc}$ or 2.8 disk-scale lengths), well within the region where in situ star formation takes place in the disk, at an approximate surface brightness of $\sim 22 \mathrm{mag} \operatorname{arcsec}^{-2}$ (3.6 $\mu \mathrm{m}$ Spitzer band). At this radius the contribution of migrated stars to either the colors, luminosity-weighted age, or chemical abundance is expected to be negligible compared with that from stars formed in situ (see Bakos et al. 2008; Vlajić et al. 2009). Again, as this brake takes place well within the star-forming disk of NGC 5668, changes in the color and metallicity gradients associated with a possible evolution of the star formation threshold with redshift is highly unlikely. With respect to the possibility that the minimum in the metal abundance profile of NGC 5668 could be due to the presence of the corotation radius at this break, two things can be said. First, NGC 5668 is a rather flocculent spiral $^{22}$ where spiral density waves are expected to be weak or absent and, consequently, the effects of the spiral arms on the radial distribution of the star formation should be minimal (if any; McCall 1986). Second, the color profiles in $(u-g)$ and $(g-r)$ show a minimum at the position of the metallicity break, which is the opposite to what we would expect if that position corresponds to the corotation radius.

\subsection{Bar Formation in NGC 5668}

The only scenario that remains to be analyzed in detail is the possibility that the deviations of the metallicity and color profiles from those predicted by the best-fitting BP2000 model of NGC 5668 are due to the presence of a nascent bar and the effects associated with it. It is well known that one of the most important drivers in the evolution of galaxies are

\footnotetext{
22 The lack of strong spiral arms in NGC 5668 is evident even after the examination of the IRAC near-infrared images of the object (see Scarano 2010 and references therein).
} 
bars (or non-axisymmetric central light distributions or ovals; Athanassoula 1994). Bars exist in about two-thirds of disk galaxies (Sellwood \& Wilkinson 1993) and isolated galaxies are known to be able to develop a barred morphology spontaneously from internal instabilities caused by cooling processes (Miller \& Prendergast 1968; Kalnajs 1978; Binney \& Tremaine 1987; Sellwood \& Wilkinson 1993). These gravitational instabilities are commonly the result of enhanced gas accretion in the disk (Lindblad et al. 1996). Some of the observational properties of NGC 5668 already reveal a potential active gas-accretion phase in this galaxy, such as the presence of both HVCs and HRVCs (Jiménez-Vicente \& Battaner 2000 and references therein) and the high total SFR in this object.

In this regard, another relevant parameter is the $\mathrm{H}$ I content of NGC 5668 compared with objects of similar total mass and morphological type. In order to find out whether the H I content NGC 5668 is particularly high or low, we resort to the so-called H I deficiency parameter. This quantity, defined by Haynes \& Giovanelli (1984), compares-in logarithmic scale-the observed $\mathrm{H}$ I mass of a given galaxy and the typical H I mass of isolated galaxies of similar morphological types and linear sizes. The difference between both values is performed in such a way that positive $\mathrm{H}$ I deficiencies correspond to galaxies with less gas than similar field galaxies, and vice versa. Following the prescriptions of Haynes \& Giovanelli (1984), we find that an Sd galaxy with the same optical diameter as NGC 5668 is expected to have $\log \left(M_{\mathrm{H}_{\mathrm{I}}}\right)=9.66$. Solanes et al. (1996) extended the work of Haynes \& Giovanelli (1984) to a larger sample of galaxies. While they only include Sa-Sc galaxies in their sample, we can safely apply the fitting coefficients of Sc's to our Sd spiral (Solanes et al. 2001). By doing so we obtain a reference $\mathrm{H}_{\mathrm{I}}$ mass of $\log \left(M_{\mathrm{HI}}\right)=9.64$, completely consistent with the previous estimation. According to Schulman et al. (1996), the actual $\mathrm{H}_{\mathrm{I}}$ mass of NGC 5668 is $\log \left(M_{\mathrm{HI}}\right)=10$, which implies a negative $\mathrm{H}$ i deficiency of $\sim-0.35$. Considering that the usual $1 \sigma$ scatter of the $\mathrm{H}$ I mass for a given type and size is $0.2-0.3 \mathrm{dex}$ (including all types of galaxies), we conclude that our Galaxy is roughly $1 \sigma-2 \sigma$ gas richer than its normal counterparts. While this difference might not be significant, it shows that, if anything, NGC 5668 has a larger H I content than the average of the spiral galaxy population of its type. Schulman et al. (1994) found in NGC 5668 a weak bar or oval inner structure of $12^{\prime \prime}$ size visible in both optical and near-infrared images. Athanassoula (1994) found that in spiral galaxies having oval structures or weak bars, star formation can occur prolifically along them, especially in the central parts and at the two ends of the bar region. This would result in bluer colors in these regions. The presence of a local minimum in color at a galactocentric distance of $\sim 30-40$ arcsec seems to favor this scenario for NGC 5668. Note, however, that the oval seen in its images is significantly smaller than the radius where the minimum in color and metallicity is found. One possible explanation for this difference might come from the fact that while the light from the superposition of $x_{1}$ orbits that shape the oval is only clearly seen in the central $12^{\prime \prime}$, the instabilities associated with it could take place further out, where the superposition of these orbits is not yet significant enough for being detected via photometry only (Debattista et al. 2006).

Another possible explanation for the existence of the oval distortion in NGC 5668 is the possible interaction of NGC 5668 with UGC9380 (Schulman et al. 1996). This is a dwarf galaxy at a relative distance of $200 \mathrm{kpc}$ to the southeast of NGC 5668, with a systemic velocity of $1690 \mathrm{~km} \mathrm{~s}^{-1}$ and a relative velocity of $\sim 108 \mathrm{~km} \mathrm{~s}^{-1}$. A recent or ongoing tidal interaction between these two galaxies could have produced the high-velocity features in NGC 5668 and triggered the formation of both the oval and the incipient bar, as reported by $N$-body simulations of minor mergers (Eliche-Moral et al. 2006, 2011).

The fact that these blue colors do not extend all the way from this galactocentric distance to the center of the galaxy is likely due to a combination of different effects. First, the secular inside-out model for the evolution of the disk of NGC 5668 predicts a reddening of $\sim 0.2 \mathrm{mag}(0.15 \mathrm{mag})$ in $u-g(g-r)$ from $36^{\prime \prime}$ to $6 "$ (see Figure 12). Moreover, the reddening in the (FUV - NUV) color indicates a change of $\sim 0.09 \mathrm{mag}$ in the $B-V$ color purely due to dust attenuation $[E(B-V)]$ within this same radial range. Finally, the light contribution of the bulge in the center, despite being small, can also lead to relatively red colors. These three effects are superimposed on the change in colors induced by star formation in the bar region and whose effects on the colors appear to be noticeable only at both its ends.

Regarding the age of this young bar, in the case of the barred galaxy NGC 3359, Martin \& Roy (1995) calculated the age of the bar by using the equation of turbulent transport in a shear flow (Roy \& Kunth 1995). Below we repeat this exercise for the case of NGC 5668. If we consider a Cartesian coordinate system centered in the galaxy center and we take $x_{2}$ as the radial direction of the local circular orbit (we do not take in account perpendicular effects), $l \sim 300 \mathrm{pc}$ as the mean free path of clouds between collisions (Roberts \& Hausman 1984), we can then calculate the time for the gas to diffuse in a length $\Delta x_{2}$ in the radial direction as

$$
\tau_{x_{2}}=\frac{\Delta x_{2}{ }^{2}}{v l} .
$$

In our case $\Delta x_{2}=4.4 \mathrm{kpc}$, which coincides with $r_{\text {BREAK }}$ which is the radius at which we detect the flattening of the metallicity gradient and the minimum in the color profiles. From the radial velocity map kindly provided by J. Jiménez-Vicente (2010, private communication; see also Jiménez-Vicente \& Battaner 2000), we determine the maximum of the non-circular motions to be $v=$ $10 \mathrm{~km} \mathrm{~s}^{-1}$. This yields $\tau_{x_{2}} \sim 10^{9} \mathrm{yr}$, which can be considered as an upper limit to the bar age since the radial transport induced by a bar is a stationary flow. The lower limit is given by a simple division of $\Delta x_{2} / v$. The best estimate for the age of the young bar in NGC 5668 is then $10^{8} \mathrm{yr} \leqslant \tau_{x_{2}} \leqslant 10^{9} \mathrm{yr}$.

In summary, while the overall observational properties of NGC 5668 are well fitted by the inside-out scenario of disk formation, the deviations in the color and metallicity profiles are best interpreted in the context of the presence of a nascent bar where significant in situ star formation is (or have been recently) taking place. The formation of the bar is believed to be due to instabilities in the gas-rich inner disk of NGC 5668, possibly helped in the interaction with a companion. This scenario is compatible with the relatively large Hi content and SFR of NGC 5668 and with the presence of HVCs and HRVCs in its velocity field, evidence of significant non-rotational gas motions in the disk.

\section{CONCLUSIONS}

In this paper, we have carried out an extensive and detailed study of the chemical and photometric properties of the nearby spiral galaxy NGC 5668. This detailed study has been possible thanks to the combined use of Integral Field Spectroscopy (IFS) 
in the optical and panchromatic broadband imaging of the entire system. The main conclusions from this work are the following.

1. Dust-attenuation profiles have been obtained using a number of methods. The mean continuum attenuation is $A_{V}$ $\sim 0.4 \mathrm{mag}$. We find a significantly larger ionized-gas attenuation than that of the continuum in agreement with the predictions of Calzetti (2001).

2. We have derived dust-attenuation-corrected emission-line fluxes for a total of 62 individual $\mathrm{H}$ II complexes and 18 concentric annuli centered on the position of the nucleus of NGC 5668. Based on the strong-line method of Kewley \& Dopita (2002) oxygen-abundance measurements have been obtained. We find a bimodal radial distribution in metallicity with a steep negative gradient with slope $-0.14 \pm$ $0.09\left(\mathrm{dex} \mathrm{kpc}^{-1}\right)$ within a galactocentric distance of $36^{\prime \prime}$ and a shallower (or even positive) metallicity gradient beyond that point, $0.002 \pm 0.002\left(\mathrm{dex} \mathrm{kpc}^{-1}\right)$.

3. Surface brightness and color profiles from the UV to the near-infrared have also been obtained. These profiles nicely match the predictions of the chemo-spectrophotometrical models for the evolution of galaxy disks (BP2000 models) for a circular velocity of $v_{\text {circ }}=167 \mathrm{~km} \mathrm{~s}^{-1}$ and a spin parameter of $\lambda=0.053$. This best-fitting model also agrees with the overall shape of the galaxy metallicity gradient and rotation curve, although it cannot reproduce the steepshallow metallicity break or the moderate bluing in some of the color profiles $(u-g, g-r)$ around the position of the metallicity break.

4. Out of the different mechanisms proposed in the literature to explain the change in the slope of the metallicity profile in spiral disks (and the color profile), only the presence of a bar in its formative stages agrees well with the other observational properties of NGC 5668: the position of the metallicity break in disk-scale lengths, the bluing in the colors at that position, the large $\mathrm{H}$ I content and SFR of the galaxy, the presence of HVCs and HRVCs in the galaxy velocity field, and even the detection of an oval in the central region of the galaxy.

We thank the anonymous referee for reviewing the manuscript, and for the comments and suggestions that helped to improve the content of the paper. We also thank Carmen ElicheMoral, Fabián Rosales-Ortega, Judit Bakos, and Sheila Kannappan for helpful discussions. R. A. Marino was also funded by the Spanish program International Campus of Excellence Moncloa (CEI). We thank the Calar Alto Observatory for the allocation of the director's discretionary time to this program. We acknowledge support from the Spanish Programa Nacional de Astronomía y Astrofísica under grant AYA 2009-10368. We are also partially funded by the Spanish MICINN under the Consolider-Ingenio 2010 Program grant CSD2006-00070: First Science with the GTC. J.C.M.M. receives financial support from NASA JPL/Spitzer grant RSA 1374189. He also acknowledges support from the National Radio Astronomy Observatory, which is a facility of the National Science Foundation operated under cooperative agreement by Associated Universities, Inc. This work is based in part on observations made with the Spitzer Space Telescope, which is operated by the Jet Propulsion Laboratory, Caltech under NASA contract 1407. GALEX is a NASA Small Explorer launched in 2003 April. We gratefully acknowledge NASA's support for construction, operation, and scientific analysis of the GALEX mission. This research has made use of the NASA/IPAC Extragalactic Database (NED) which is operated by the Jet Propulsion Laboratory, and the California Institute of Technology, under contract with the National Aeronautics and Space Administration. This paper makes use of the Sloan Digital Sky Survey data. Funding for the SDSS and SDSS-II has been provided by the Alfred P. Sloan Foundation, the Participating Institutions, the National Science Foundation, the U.S. Department of Energy, the National Aeronautics and Space Administration, the Japanese Monbukagakusho, the Max Planck Society, and the Higher Education Funding Council for England. The SDSS Web site is http://www.sdss.org/. The SDSS is managed by the Astrophysical Research Consortium for the Participating Institutions. The Participating Institutions are the American Museum of Natural History, the Astrophysical Institute Potsdam, the University of Basel, the University of Cambridge, Case Western Reserve University, the University of Chicago, Drexel University, Fermilab, the Institute for Advanced Study, the Japan Participation Group, Johns Hopkins University, the Joint Institute for Nuclear Astrophysics, the Kavli Institute for Particle Astrophysics and Cosmology, the Korean Scientist Group, the Chinese Academy of Sciences (LAMOST), the Los Alamos National Laboratory, the MaxPlanck-Institute for Astronomy (MPIA), the Max-PlanckInstitute for Astrophysics (MPA), New Mexico State University, the Ohio State University, the University of Pittsburgh, the University of Portsmouth, Princeton University, the United States Naval Observatory, and the University of Washington.

\section{REFERENCES}

Abazajian, K. N., Adelman-McCarthy, J. K., Agüeros, M. A., et al. 2009, ApJS, 182,543

Acharova, I. A., Lépine, J. R. D., \& Mishurov, Y. N. 2005, MNRAS, 359, 819 Ardeberg, A., \& Virdefors, B. 1982, A\&A, 115, 347

Athanassoula, E. 1994, in Proc. of the Conf. held at The University of Kentucky, Lexington, ed. I. Shlosman (Cambridge: Cambridge Univ. Press), 143

Azzollini, R., Trujillo, I., \& Beckman, J. E. 2008, ApJ, 679, L69

Bakos, J., Trujillo, I., \& Pohlen, M. 2008, ApJ, 683, L103

Barbanis, B., \& Woltjer, L. 1967, ApJ, 150, 461

Barden, M., Rix, H., Somerville, R. S., et al. 2005, ApJ, 635, 959

Barker, M. K., Ferguson, A. M. N., Irwin, M., Arimoto, N., \& Jablonka, P. 2009, AJ, 138, 1469

Bell, E. F. 2002, ApJ, 577, 150

Bell, E. F., \& de Jong, R. S. 2000, MNRAS, 312, 497

Binney, J. 2001, in Galaxy Disks and Disk Galaxies, ed. J. G. Funes, S. J., \& E. M. Corsini (San Francisco, CA: ASP), 63

Binney, J., \& Tremaine, S. 1987, Galactic Dynamics (Princeton, NJ: Princeton Univ. Press)

Boffi, F. R., Sparks, W. B., \& Macchetto, F. D. 1999, A\&AS, 138, 253

Boissier, S., Gil de Paz, A., Boselli, A., et al. 2007, ApJS, 173, 524

Boissier, S., \& Prantzos, N. 1999, MNRAS, 307, 857

Boissier, S., \& Prantzos, N. 2000, MNRAS, 312, 398

Boselli, A., Eales, S., Cortese, L., et al. 2010, PASP, 122, 261

Bresolin, F., Gieren, W., Kudritzki, R., et al. 2009a, ApJ, 700, 309

Bresolin, F., Kennicutt, R. C., \& Ryan-Weber, E. 2012, ApJ, 750, 122

Bresolin, F., Ryan-Weber, E., Kennicutt, R. C., \& Goddard, Q. 2009b, ApJ, 695, 580

Bruzual, G., \& Charlot, S. 2003, MNRAS, 344, 1000

Buat, V. 1992, A\&A, 264, 444

Buat, V., Iglesias-Páramo, J., Seibert, M., et al. 2005, ApJ, 619, L51

Calzetti, D. 1997, AJ, 113, 162

Calzetti, D. 2001, PASP, 113, 1449

Calzetti, D., Armus, L., Bohlin, R. C., et al. 2000, ApJ, 533, 682

Calzetti, D., Kinney, A. L., \& Storchi-Bergmann, T. 1994, ApJ, 429, 582

Calzetti, D., Kinney, A. L., \& Storchi-Bergmann, T. 1996, ApJ, 458, 132

Cardelli, J. A., Clayton, G. C., \& Mathis, J. S. 1989, ApJ, 345, 245

Charlot, S., \& Fall, S. M. 2000, ApJ, 539, 718

Chiappini, C., Matteucci, F., \& Romano, D. 2001, ApJ, 554, 1044

Considère, S., Coziol, R., Contini, T., \& Davoust, E. 2000, A\&A, 356, 89

Cortese, L., Boselli, A., Buat, V., et al. 2006, ApJ, 637, 242

Cortese, L., Boselli, A., Franzetti, P., et al. 2008, MNRAS, 386, 1157

Dale, D. A., Gil de Paz, A., Gordon, K. D., et al. 2007, ApJ, 655, 863 
Debattista, V. P., Mayer, L., Carollo, C. M., et al. 2006, ApJ, 645, 209 de Jong, R. S., Seth, A. C., Radburn-Smith, D. J., et al. 2007, ApJ, 667, L49 de Vaucouleurs, G., de Vaucouleurs, A., Corwin, H. G., Jr., et al. (ed.) 1991, Third Reference Catalogue of Bright Galaxies (New York: Springer)

Díaz, A. I., Terlevich, E., Pagel, B. E. J., Vílchez, J. M., \& Edmunds, M. G. 1990, RevMexAA, 21, 223

Dressel, L. L., \& Condon, J. J. 1976, ApJS, 31, 187

Edmunds, M. G., \& Roy, J. 1993, MNRAS, 261, L17

Eliche-Moral, M. C., Balcells, M., Aguerri, J. A. L., \& González-García, A. C. 2006, A\&A, 457, 91

Eliche-Moral, M. C., González-García, A. C., Balcells, M., et al. 2011, A\&A, 533, A104

Epinat, B., Amram, P., \& Marcelin, M. 2008, MNRAS, 390, 446

Fazio, G. G., Hora, J. L., Allen, L. E., et al. 2004, ApJS, 154, 10

Fitzpatrick, E. L. 1999, PASP, 111, 63

Fixsen, D. J., Cheng, E. S., Gales, J. M., et al. 1996, ApJ, 473, 576

Friedli, D., \& Benz, W. 1995, A\&A, 301, 649

Friedli, D., Benz, W., \& Kennicutt, R. 1994, ApJ, 430, L105

Fuchs, B. 2001, MNRAS, 325, 1637

Garnett, D. R. 1992, AJ, 103, 1330

Gil de Paz, A., Boissier, S., Madore, B. F., et al. 2007, ApJS, 173, 185

Gil de Paz, A., \& Madore, B. F. 2002, AJ, 123, 1864

Gil de Paz, A., \& Madore, B. F. 2005, ApJS, 156, 345

Gil de Paz, A., Madore, B. F., Boissier, S., et al. 2005, ApJ, 627, L29

Gogarten, S. M., Dalcanton, J. J., Williams, B. F., et al. 2010, ApJ, 712, 858

Gordon, K. D., Clayton, G. C., Witt, A. N., \& Misselt, K. A. 2000, ApJ, 533, 236

Haynes, M. P., \& Giovanelli, R. 1984, AJ, 89, 758

Haywood, M. 2008, MNRAS, 388, 1175

Heckman, T., Krolik, J., Meurer, G., et al. 1995, ApJ, 452, 549

Jensen, E. B., Strom, K. M., \& Strom, S. E. 1976, ApJ, 209, 748

Jiménez-Vicente, J., \& Battaner, E. 2000, A\&A, 358, 812

Kalnajs, A. J. 1978, in Structure and Properties of Nearby Galaxies (IAU Symp.

77), ed. E. M. Berkhuisjen \& R. Wielebinski (Dordrecht: Reidel), 113

Kelz, A., Verheijen, M. A. W., Roth, M. M., et al. 2006, PASP, 118, 129

Kewley, L. J., \& Dopita, M. A. 2002, ApJS, 142, 35

Kroupa, P. 2001, MNRAS, 322, 231

Lindblad, P. A. B., Lindblad, P. O., \& Athanassoula, E. 1996, A\&A, 313, 65

MacArthur, L. A., Courteau, S., Bell, E., \& Holtzman, J. A. 2004, ApJS, 152, 175

Maíz-Apellániz, J., Mas-Hesse, J. M., Muñoz-Tuñon, C., Vílchez, J. M., \& Castañeda, H. O. 1998, A\&A, 329, 409

Maraston, C., Daddi, E., Renzini, A., et al. 2006, ApJ, 652, 85

Mármol-Queraltó, E., Sánchez, S. F., Marino, R. A., et al. 2011, A\&A, 534, A8

Martin, C. L., \& Kennicutt, R. C., Jr. 2001, ApJ, 555, 301

Martin, D. C., Fanson, J., Schiminovich, D., et al. 2005, ApJ, 619, L1

Martin, P., \& Roy, J. 1995, ApJ, 445, 161

Mayya, Y. D., \& Prabhu, T. P. 1996, AJ, 111, 1252

McCall, M. L. 1986, PASP, 98, 992

McCall, M. L., Rybski, P. M., \& Shields, G. A. 1985, ApJS, 57, 1

McGaugh, S. S. 1991, ApJ, 380, 140

Meurer, G. R., Heckman, T. M., \& Calzetti, D. 1999, ApJ, 521, 64

Meurer, G. R., Heckman, T. M., Leitherer, C., et al. 1995, AJ, 110, 2665

Miller, R. H., \& Prendergast, K. H. 1968, ApJ, 151, 699

Minchev, I., Famaey, B., Combes, F., et al. 2011, A\&A, 527, A147

Mo, H. J., Mao, S., \& White, S. D. M. 1998, MNRAS, 295, 319

Morrissey, P., Conrow, T., Barlow, T. A., et al. 2007, ApJS, 173, 682

Moustakas, J., Kennicutt, R. C., Jr., Tremonti, C. A., et al. 2010, ApJS, 190, 233

Muñoz-Mateos, J. C., Boissier, S., Gil de Paz, A., et al. 2011, ApJ, 731, 10

Muñoz-Mateos, J. C., Gil de Paz, A., Boissier, S., et al. 2007, ApJ, 658, 1006

Muñoz-Mateos, J. C., Gil de Paz, A., Boissier, S., et al. 2009a, ApJ, 701, 1965

Muñoz-Mateos, J. C., Gil de Paz, A., Zamorano, J., et al. 2009b, ApJ, 703, 1569

Nakano, S., Kushida, R., Kushida, Y., \& Itagaki, K. 2004, IAU Circ., 8272, 1

Oke, J. B. 1990, AJ, 99, 1621
Oort, A. H., \& Peixóto, J. P. 1974, J. Geophys. Res., 79, 2705

Osterbrock, D. E., \& Ferland, G. J. 2006, Astrophysics of Gaseous Nebulae and Active Galactic Nuclei (2nd. ed; Sausalito, CA: University Science Books)

Pagel, B. E. J. 1986, PASP, 98, 1009

Pagel, B. E. J., Edmunds, M. G., Blackwell, D. E., Chun, M. S., \& Smith, G. 1979, MNRAS, 189, 95

Pérez-Montero, E., \& Díaz, A. I. 2005, MNRAS, 361, 1063

Poggianti, B. M., Smail, I., Dressler, A., et al. 1999, ApJ, 518, 576

Poggianti, B. M., \& Wu, H. 2000, ApJ, 529, 157

Pohlen, M., \& Trujillo, I. 2006, A\&A, 454, 759

Prantzos, N., \& Boissier, S. 2000, MNRAS, 313, 338

Reach, W. T., Megeath, S. T., Cohen, M., et al. 2005, PASP, 117, 978

Roberts, W. W., Jr., \& Hausman, M. A. 1984, ApJ, 277, 744

Rosales-Ortega, F. F. 2011, NewA, 16, 220

Rosales-Ortega, F. F., Kennicutt, R. C., Sánchez, S. F., et al. 2010, MNRAS, 405, 735

Roškar, R., Debattista, V. P., Loebman, S. R., Ivezić, Ž., \& Quinn, T. R. 2011, MNRAS, submitted (arXiv:1110.4413)

Roškar, R., Debattista, V. P., Quinn, T. R., Stinson, G. S., \& Wadsley, J. 2008, ApJ, 684, L79

Roy, J., Belley, J., Dutil, Y., \& Martin, P. 1996, ApJ, 460, 284

Roy, J., \& Kunth, D. 1995, A\&A, 294, 432

Roy, J., \& Walsh, J. R. 1997, MNRAS, 288, 715

Sánchez, S. F. 2004, Astron. Nachr., 325, 167

Sánchez, S. F. 2006, Astron. Nachr., 327, 850

Sánchez-Blázquez, P., Courty, S., Gibson, B. K., \& Brook, C. B. 2009, MNRAS, 398, 591

Sánchez-Blázquez, P., Marcolini, A., Gibson, B. K., et al. 2012, MNRAS, 419, 1376

Sánchez-Blázquez, P., Peletier, R. F., Jiménez-Vicente, J., et al. 2006, MNRAS, 371,703

Sandage, A., \& Tammann, G. A. 1987, A Revised Shapley-Ames Catalog of Bright Galaxies (Carnegie Inst. Washington Publ., Washington, D.C.: Carnegie Inst. Washington)

Savage, B. D., \& Mathis, J. S. 1979, ARA\&A, 17, 73

Scarano, S., Jr. 2010, Bull. Astron. Soc. Braz., 29, 65

Schulman, E., Bregman, J. N., Brinks, E., \& Roberts, M. S. 1996, AJ, 112, 960

Schulman, E., Bregman, J. N., \& Roberts, M. S. 1994, ApJ, 423, 180

Seaton, M. J. 1979, MNRAS, 187, 73P

Seibert, M., Martin, D. C., Heckman, T. M., et al. 2005, ApJ, 619, L55

Sellwood, J. A., \& Binney, J. J. 2002, MNRAS, 336, 785

Sellwood, J. A., \& Wilkinson, A. 1993, Rep. Prog. Phys., 56, 173

Shaver, P. A., McGee, R. X., Newton, L. M., Danks, A. C., \& Pottasch, S. R. 1983, MNRAS, 204, 53

Solanes, J. M., Giovanelli, R., \& Haynes, M. P. 1996, ApJ, 461, 609

Solanes, J. M., Manrique, A., García-Gómez, C., et al. 2001, ApJ, 548, 97

Spitzer, L., Jr., \& Schwarzschild, M. 1953, ApJ, 118, 106

Stasińska, G., \& Sodré, L., Jr. 2001, A\&A, 374, 919

Thilker, D. A., Bianchi, L., Meurer, G., et al. 2007, ApJS, 173, 538

Thurston, T. R., Edmunds, M. G., \& Henry, R. B. C. 1996, MNRAS, 283, 990

Trujillo, I., Förster Schreiber, N. M., Rudnick, G., et al. 2006, ApJ, 650, 18

Trujillo, I., Rudnick, G., Rix, H., et al. 2004, ApJ, 604, 521

Trumpler, R. J. 1930, PASP, 42, 214

Vila-Costas, M. B., \& Edmunds, M. G. 1992, MNRAS, 259, 121

Vílchez, J. M., \& Esteban, C. 1996, MNRAS, 280, 720

Vlajić, M., Bland-Hawthorn, J., \& Freeman, K. C. 2009, ApJ, 697, 361

Wang, L., \& Rowan-Robinson, M. 2009, MNRAS, 398, 109

Werner, M. W., Roellig, T. L., Low, F. J., et al. 2004, ApJS, 154, 1

Witt, A. N., \& Gordon, K. D. 2000, ApJ, 528, 799

Xu, C., \& Buat, V. 1995, A\&A, 293, L65

Yoachim, P., \& Dalcanton, J. J. 2008, ApJ, 682, 1004

Yoachim, P., Roškar, R., \& Debattista, V. P. 2010, ApJ, 716, L4

York, D. G., Adelman, J., Anderson, J. E., Jr., et al. 2000, AJ, 120, 1579

Zaritsky, D., Kennicutt, R. C., Jr., \& Huchra, J. P. 1994, ApJ, 420, 87 